\title{
THE EVALUATION OF ECOSYSTEM HEALTH BASED ON HYBRID TODIM METHOD FOR CHINESE CASE
}

\author{
Peide LIU ${ }^{1^{*}}$, Lili RONG ${ }^{1,2}$, Fei TENG ${ }^{1}$ \\ ${ }^{1}$ School of Management Science and Engineering, Shandong University of Finance and Economics, \\ Jinan Shandong 250014, China \\ ${ }^{2}$ School of Computer and Communication Engineering, Shandong TV University, \\ Jinan Shandong 250014, China
}

Received 17 May 2018; accepted 25 November 2018

\begin{abstract}
The health evaluation of urban ecosystem is the need of urban sustainable development and the construction of urban ecological civilization, in order to scientifically evaluate the ecosystem health, in this paper, we establish the mathematical model based on hybrid multiple attributes decision-making. Firstly, we introduce the original city ecosystem health evaluation indexes which reflect on Vitality, Composition Structure, Recovery Capacity, The Ecological System Continually Offering Service Function, Population Health and Ecosystem Cognition ecosystem health. Then in order to obtain the reasonable weights, we integrate the subjective weights by linguistic AHP method and objective weights by deviation maximization method, and get the combined weights for city ecosystem health evaluation indexes. Further, according to the characteristics of the different indexes, we propose an extended TODIM method to evaluate the city ecosystem health in which the indexes take the form of real number, interval number, and probabilistic linguistic term set. Moreover, with respect to the evaluation values of city ecosystem health in Jinan from 2011 to 2015 , this paper evaluates the health status of Jinan ecological system, and analyzes the role of various indicators in the process of city ecological development. Result shows that: (1) Jinan ecosystem health status remained at the sub-health state from 2011 to 2015, and the ecological situation is not optimistic. (2) Prominent problems restricting are lack of investment in environmental protection efforts, increasing pollutant emissions, and imperfect industrial structure. To solve the problems in the healthy development of Jinan urban ecosystem, this paper puts forward corresponding countermeasures and suggestions to improve the healthy development of urban ecosystem.
\end{abstract}

Keywords: urban ecosystem health, extended TODIM method, hybrid information, combined weighted method.

JEL Classification: C15, C44, C80.

${ }^{\star}$ Corresponding author. E-mail: peide.liu@gmail.com

This is an Open Access article distributed under the terms of the Creative Commons Attribution License (http://creativecommons. $\mathrm{org} / \mathrm{licenses} / \mathrm{by} / 4.0 /$ ), which permits unrestricted use, distribution, and reproduction in any medium, provided the original author and source are credited. 


\section{Introduction}

Since the beginning of 21st century, the urbanization of China has become the mainstream of world urban development in the background of globalization. The urban ecosystem environment has been seriously threatened (Brevik et al., 2019; du Preez, Daneel, Wepener, \& Fourie, 2018; van Heezik \& Brymer, 2018). Urban ecosystem is a complex ecosystem, which is characterized by vulnerability, complexity and dependence. It is highly susceptible for human or natural environment. As a material basis for human survival and development, a healthy ecosystem is the fundamental guarantee for the sustainable development of society and economy, and the basic condition for human survival.

Various countries and governments have paid more attention to the problem of urban ecological environment, and many scientific researches about ecosystem health have been carried out. In the 1990s, the concept of sustainable development had become the research focus in academia and the government. It is in this concept that the ecosystem health research becomes the new research hot topic (Brussard, Reed, \& Tracy, 1998; Haeuber, 1998; Lackey, 1998). After those researches, Gentile et al. (2001), Berberoglu (2003) and Noss (2000) assessed the ecological risks of southern Florida, Turkey's eastern Mediterranean coast, and Quebec, Canada. In order to understand the health condition of the ecosystem health, the health status of ecosystem needs to be scientifically evaluated and analyzed. In the decade after 2000, many experts and scholars have done a lot of researches on ecosystem health evaluation index system. Bell et al. (2011) established the environmental health system of five indicators for air pollution and health for urban Latin American centers. Y. He, Hao, W. He, Lam, and Xu (2019) evaluated the changes of ecosystem in Hong Kong waters in accordance with monthly data from 1986 to 2014. Derkzen, Teeffelen, and Verburg (2015) evaluated the ecosystem services of Rotterdam, Netherlands, combined with different methods and evidences. Although the above researches have made a progress on evaluation index system, those on urban ecosystem health are not profound. Many scholars (Spiegel et al., 2001; Bayram, Önsoy, Bulut, \& Akinci, 2013; Li, Ye, Song, \& Wang, 2014) constructed the evaluation index systems of city ecosystem health, and ecosystem health of Havana (Cuba), Gumushane (Turkey), and Changzhou (China) was evaluated respectively, which found out the influence factors of local city ecosystem health, then put forward the solutions.

In addition, a number of multi-criteria decision-making models and methods can also be used for ecological environmental assessment (Li \& Liu, 2015; Liu, 2017; Liu \& Chen, 2017; Liu \& Chen, 2018; P. Liu; Chen, \& J. Liu, 2017a; Liu \& Li, 2017; P. Liu, J. Liu, \& Chen, 2018a; P. Liu, J. Liu, \& Merigó, 2018b; Liu \& Wang, 2018). In these processes of evaluation, the information generally adopts the form of real number, but in real life, it is often described with hybrid variables. Guan, Sun, and Zhao (2016) applied extended VIKOR method to the ecosystem health evaluation in Yellow River Belt, in which the evaluation index can take the form of the real number, the interval number, the linguistic variable and two-dimensional linguistic variable. Mahdi, Hosseini, Pourahmad, and Hataminejad (2016) applied the TOPSIS method to the health status of city ecological system in Qom. However, VIKOR and TOPSIS methods may influence the consequence results due to the improper selection of ideal solution. Different measure definitions and relative proximity function may get different results. 
TODIM (an acronym in Portuguese of interactive and multi-criteria decision making) is a multi attribute decision making (MADM) method, which needn't select positive or negative ideal solution and can avoid the inaccurate result (Gomes \& Lima, 1991; Llamazares, 2018; Wu, Wang, Hu, Ke, \& Li, 2018; Mishra \& Rani, 2018; Huang \& Wei, 2018; Huang, Li, \& Liu, 2017; Lourenzutti, Krohling, \& Reformat, 2017; Jiang, X. Liang, \& H. Liang, 2017). With the wide application in real life, it is designed to help people make effective evaluation, and to solve the defects of TOPSIS method and VIKOR method in the decision-making process. Then TODIM method has been applied widely (Pereira, 2013; Salomon \& Rangel, 2015; Gomes \& Rangel, 2009). Although classical TODIM is applied better than VIKOR and TOPSIS in solving MADM problems, it can only handle the format of crisp number (Fan, Zhang, Chen, \& Liu, 2013). Urban ecosystem evaluation concerns more than the format of crisp numbers, which depends on various formats of attribute values. According to the above, extended TODIM method should be further applied to solve hybrid MADM problem, including the form of real number, interval number, and probabilistic linguistic term set.

Therefore, this paper uses the extended TODIM method based on prospect theory to evaluate the health status of Jinan city ecosystem, and uses the health index method to analyze change trend of each indicator, so as to find out the adverse health indicators of the development process of Jinan city in the ecological system. According to the research conclusion and the actual situation of Jinan, the corresponding countermeasures are given in order to realize the healthy development of urban ecological system in Jinan.

The structure of this paper is organized as follows: in Section 1, the calculation model is established, which contains normalization of evaluation information, the distance formula of the evaluation value of hybrid index, and combined weighted method of different indicator. Section 2 introduces extended TODIM method of hybrid information. In Section 3, an empirical study of ecosystem health assessment in Jinan is described. In Section 4, the changes of urban ecosystem health in Jinan are analyzed. In final section, the conclusion is given.

\section{The calculation model of index weights on urban ecological system health evaluation}

\subsection{Normalization of evaluation information}

In order to eliminate the influence of following differences, the incommensurability and contradiction between different attribute types, and the different dimensions and magnitudes, the original information $X=\left[x_{i j}\right]_{m \times n}$ (let $m$ be the number of evaluation alternatives, and $n$ be the number of indicator, $x_{i j}$ be the evaluation value of alternative $i$ under the indicator $j$ ) should be normalized. In this paper, the evaluation of hybrid indicator (the real numbers, the interval numbers and probabilistic linguistic term sets) is discussed, the normalized matrix is $B=\left[b_{i j}\right]_{m \times n}$, the normalized method is defined as follows $\left(j \in I_{1}\right.$ expresses benefit type indicator and $j \in I_{2}$ cost type indicator).

(1) The normalized method for real numbers is shown as follows (Liu, 2009):

$$
b_{i j}=x_{i j} / \sqrt{\sum_{i=1}^{m} x_{i j}^{2}} \quad j=I_{1} ;
$$




$$
b_{i j}=1 / x_{i j} / \sqrt{\sum_{i=1}^{m}\left(1 / x_{i j}\right)^{2}} \quad j \in I_{2},
$$

$b_{j}$ is the normalized real number, $x_{i j}$ is the original real number, $I_{1}$ is a set of benefit indicators and $I_{2}$ is a set of cost indicators.

(2) The normalized method of interval numbers is shown as follows (Liu, 2009):

Let $x_{i j}=\left[a_{i j}^{l}, a_{i j}^{r}\right]$ be interval number, the normalized number is $b_{i j}=\left[b_{i j}^{l}, b_{i j}^{r}\right]$ :

$$
\begin{aligned}
& \left\{\begin{array}{l}
b_{i j}^{l}=a_{i j}^{l} / \sqrt{\sum_{i=1}^{m}\left[\left(a_{i j}^{l}\right)^{2}+\left(a_{i j}^{r}\right)^{2}\right]} \\
b_{i j}^{r}=a_{i j}^{r} / \sqrt{\sum_{i=1}^{m}\left[\left(a_{i j}^{l}\right)^{2}+\left(a_{i j}^{r}\right)^{2}\right]} \quad j \in I_{1} ;
\end{array}\right. \\
& \left\{\begin{array}{l}
b_{i j}^{l}=1 / a_{i j}^{r} / \sqrt{\sum_{i=1}^{m}\left[\left(1 / a_{i j}^{l}\right)^{2}+\left(1 / a_{i j}^{r}\right)^{2}\right]} \\
b_{i j}^{r}=1 / a_{i j}^{l} / \sqrt{\sum_{i=1}^{m}\left[\left(1 / a_{i j}^{l}\right)^{2}+\left(1 / a_{i j}^{r}\right)^{2}\right]}
\end{array} \in I_{2},\right.
\end{aligned}
$$

$a_{i j}^{l}, a_{i j}^{r}$ are respectively the lower limit and upper limit of the original interval number, $b_{i j}^{l}, b_{i j}^{r}$ are respectively the lower limit and upper limit of the normalized interval number, $I_{1}$ is a set of benefit indicators and $I_{2}$ is a set of cost indicators.

(3) The normalized method of probabilistic linguistic term set (PLTS) is shown as follows (Pang, Wang, \& Xu, 2016):

Let $L(p)=\left\{L^{(k)}\left(p^{(k)}\right) \mid L^{(k)} \in S, p^{(k)} \geq 0, k=1,2, \ldots, \# L(p), \sum_{k=1}^{\# L(p)} p^{(k)} \leq 1\right\}$ be a PLTS,

where $L^{(k)}\left(p^{(k)}\right)$ is the linguistic term $L^{(k)}$ associated with the probability $p^{(k)}$, and $\# L(p)$ is the number of all different linguistic terms in $L(p)$. The normalized PLTS is

$$
\tilde{L}_{i j}(p)=\left\{\begin{array}{ll}
L_{i j}(p), & j \in I_{1} \\
\left(L_{i j}(p)\right)^{c}, & j \in I_{2}
\end{array},\right.
$$

where $I_{1}$ is a set of benefit indicator and $I_{2}$ is a set of cost indicator $\left(L_{i j}(p)\right)^{c}$ is complement of $L_{i j}(p), L_{i j}^{c}(p)=\left\{n e g\left(L_{i j}^{(k)}\right)\left(p_{i j}^{(k)}\right) \mid k=1,2, \ldots, \# L_{i j}(p)\right\}$.

\subsection{The distance formula of the evaluation value of hybrid index}

Based on the normalized information, the distance measure $d\left(b_{i j}, b_{k j}\right)$ of different schemes under certain indicator is calculated as following:

(1) Distance measure between real numbers is

$$
d\left(b_{i j}, b_{k j}\right)=\left|b_{i j}-b_{k j}\right|
$$


where $d\left(b_{i j}, b_{k j}\right)$ is the distance between the real numbers $b_{i j}$ and $b_{k j}$.

(2) Distance measure between interval numbers is

$$
d\left(b_{i j}, b_{k j}\right)=\frac{\sqrt{2}}{2} \sqrt{\left(b_{i j}{ }^{l}-b_{k j}{ }^{l}\right)^{2}+\left(b_{i j}{ }^{r}-b_{k j}{ }^{r}\right)^{2}},
$$

where $d\left(b_{i j}, b_{k j}\right)$ is the distance between the interval numbers $b_{i j}$ and $b_{k j} ; b_{i j}^{l}, b_{i j}^{r}$ are respectively the lower limit and upper limit of the normalized interval number $b_{i j} ; b_{k j}^{l}, b_{k j}^{r}$ are respectively the lower limit and upper limit of the normalized interval number $b_{k j}$.

(3) Distance measure of probabilistic linguistic term set (PLTS) is (Pang et al., 2016).

Let $L_{i j}(p)=\left\{L_{i j}^{\left(d_{1}\right)}\left(p_{i j}^{\left(d_{1}\right)}\right) \mid d_{1}=1,2, \ldots, \# L_{i j}(p)\right\}$ and

$L_{k j}(p)=\left\{L_{k j}^{\left(d_{2}\right)}\left(p_{k j}^{\left(d_{2}\right)}\right) \mid d_{2}=1,2, \ldots, \# L_{k j}(p)\right\}$ be two PLTSs, \# $L_{i j}(p)=\# L_{k j}(p)$.

Then the distance between $L_{i j}(p)$ and $L_{k j}(p)$ is defined as:

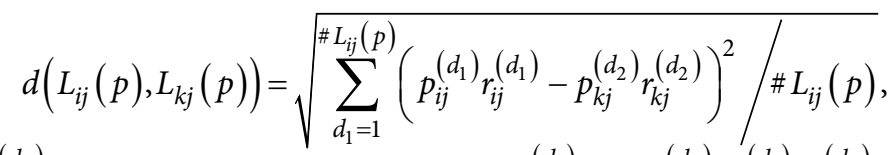

where $r_{i j}^{\left(d_{1}\right)}, r_{k j}^{\left(d_{2}\right)}$ are respectively the subscripts of $L_{i j}^{\left(d_{1}\right)}$ and $L_{k j}^{\left(d_{2}\right)} ; p_{i j}^{\left(d_{1}\right)}, p_{k j}^{\left(d_{2}\right)}$ are respectively the possibilities of $L_{i j}^{\left(d_{1}\right)}$ and $L_{k j}^{\left(d_{2}\right)}$.

\subsection{Combined weighted method of different indicators}

The AHP method depends on the experience of the decision-maker and the subjective importance of evaluation index, which is an effective method of subjective empowerment. The dispersion maximization method is an objective weighting method, which makes full use of the decision data and shows certain objectivity. To present both subjective weights and objective weights, the combined weighted method is the appropriate one. The linear weighted method is an effective combined weighted method which can adjust the proportion of the subjective and objective weight according to the preference of the decision maker with great flexibility.

In order to directly use linguistic comparison information, we adopt the linguistic AHP method which can avoid the problem that the gap between the maximum and the minimum is too large (Liu, 2009).

Firstly, based on the linguistic decision matrix $A=\left(a_{i j}\right)_{n \times n}$ by experts, the matrix $R=\left(r_{i j}\right)_{n \times n}$ is constructed:

$$
r_{i j}=\exp \left(\frac{I\left(a_{i j}\right)-g}{g}\right), a_{i j} \in S, i, j=1,2, \cdots, t,
$$

$a_{i j}$ is the linguistic variable for importance of index $i$ with respect to index $j$ given by experts, and $I\left(a_{i j}\right)$ is the subscript of the linguistic term, i.e., If $a_{i j}=s_{k}$, then $I\left(a_{i j}\right)=k, g=l-1 / 2$, $l$ is the number of elements in the linguistic term set $S$.

Based on the formula (9), the subjective weight $\zeta_{i}$ is calculated:

$$
S_{i}=\sqrt[n]{\prod_{j=1}^{n} r_{i j}} / \sum_{i=1}^{n} \sqrt[n]{\prod_{j=1}^{n} r_{i j}}
$$


Secondly, the objective weight $w_{j}$ is calculated:

$$
w_{j}=\sum_{i=1}^{m} \sum_{k=1}^{m} d\left(b_{i j}, b_{k j}\right) / \sum_{j=1}^{n} \sum_{i=1}^{m} \sum_{k=1}^{m} d\left(b_{i j}, b_{k j}\right),
$$

$d\left(b_{i j}, b_{k j}\right)$ is the distance measure of $b_{i j}, b_{k j}$.

Finally, based on the subjective weight and objective weight, the combination weight $\omega_{j}$ of each indicator is calculated:

$\omega_{j}=\alpha \times \varsigma_{j}+\beta \times w_{j}$,
$\varsigma_{j}$ is subjective weight and $\sum_{j=1}^{n} \varsigma_{j}=1,0 \leq \varsigma_{j} \leq 1, w_{j}$ is the objective weight and $\sum_{j=1}^{n} w_{j}=1$, $0 \leq w_{j} \leq 1, \alpha$ and $\beta$ are two parameters, which satisfies $\alpha+\beta=1, \alpha \geq 0, \beta \geq 0$.

\section{A model of urban ecosystem health evaluation based on the extended TODIM method of hybrid information}

In the urban ecosystem health evaluation, based on 34 indicators $C=\left(C_{1}, C_{2}, \cdots, C_{34}\right)$ this paper evaluates the ecosystem health $A=\left(A_{1}, A_{2}, \cdots, A_{5}\right)$ between 2011 and 2015, in this process of evaluation, evaluation matrix is $X=\left[x_{i j}\right]_{m \times n}$, where $x_{i j}$ is the evaluation value of alternative $A_{i}$ with respect to indicator $C_{j}, x_{i j}$ can be expressed by the real number, the interval number and probability linguistic term set. In accordance with this situation, this paper puts forward a model of urban ecosystem health evaluation based on the extended TODIM method of hybrid information, and the whole process is shown as follows:

Step 1: Normalize the original decision matrix $X=\left[x_{i j}\right]_{m \times n}$ into the normalized matrix $B=\left[b_{i j}\right]_{m \times n}$ in accordance with section 1.1.

Step 2: Calculate the combination weight $\omega=\left(\omega_{1}, \omega_{2}, \ldots, \omega_{n}\right)$ of the indicator in accordance with section 1.3 .

Step 3: Determine the relative weight of indicator by the following formula.

$$
\omega_{j}^{\prime}=\frac{\omega_{j}}{\omega^{*}}, j=1,2, \ldots, n,
$$

where $\omega^{*}=\max \left\{\omega_{1}, \omega_{2}, \ldots, \omega_{n}\right\}$ is reference weight.

Step 4: Calculate the relative dominance of each alternative $A_{i}$ over $A_{k}$ under each indicator

$$
\phi_{j}\left(A_{i}, A_{k}\right)= \begin{cases}\sqrt{\frac{\omega_{j}^{\prime}}{\sum_{j=1}^{n} \omega_{j}^{\prime}} d\left(b_{i j}, b_{k j}\right)} & b_{i j}>b_{k j} \\ 0 & b_{i j}=b_{k j}, \\ -\frac{1}{\theta} \sqrt{\frac{\sum_{j=1}^{n} \omega_{j}^{\prime}}{\omega_{j}^{\prime}} d\left(b_{i j}, b_{k j}\right)} & b_{i j}<b_{k j}\end{cases}
$$

where $\theta$ is the attenuation factor of the losses. The smaller the $\theta$, the higher the loss avoid- 
ance of decision makers. If $\theta<1$, then the impact of losses will intensify; and if $\theta>1$, then the impact of losses will be reduced. $\theta=1$ and $\theta=2.5$ are the common parameter values. $d\left(b_{i j}, b_{k j}\right)$ represents the distance between the two values.

Step 5: Calculate the overall dominance of the alternative

$$
\delta\left(A_{i}, A_{k}\right)=\sum_{j=1}^{n} \phi_{j}\left(A_{i}, A_{k}\right) .
$$

Step 6: Calculate the overall prospect value of each alternative $A_{i}$

$$
\xi_{i}=\frac{\sum_{k=1}^{m} \delta\left(A_{i}, A_{k}\right)-\min _{i} \sum_{k=1}^{m} \delta\left(A_{i}, A_{k}\right)}{\max _{i} \sum_{k=1}^{m} \delta\left(A_{i}, A_{k}\right)-\min _{i} \sum_{k=1}^{m} \delta\left(A_{i}, A_{k}\right)} .
$$

Step 7: Rank possible alternatives according to the prospect values $\xi_{i}$.

\section{An empirical study of ecosystem health assessment in Jinan}

Jinan is the capital of Shandong province (the largest coastal province in eastern China). As important transportation hub of the provincial political, economic, cultural, technological, educational and financial center, Jinan, the capital economic circle, connected to Shandong Peninsula and the central region, is the important intersection of Bohai economic zone and Beijing Shanghai economy on the axis of Jinan. With the development of economic and urbanization, the ecosystem health has been worsened. The period between 2011 and 2015 is the twelfth five-year plan for China's economic and social development. Although Jinan government made great effort to carry out a series of reform, according to Jinan statistical yearbook (2012-2016), the average annual concentration of PM10 and SO2 exceeded the air quality standard; economic structure and energy structure are unreasonable, structural pollution is serious. Therefore, the quality of ecological environment needs improving.

In order to evaluate the ecosystem health in Jinan, we need build the ecosystem health evaluation index. For convenience, we adopt the following health evaluation index of urban ecosystem in Jinan from Liu, Teng, Chu, and Guan (2017b) shown in Table 1.

\subsection{The determination of the subjective weights of indicators}

The combined weight of Jinan urban ecosystem health evaluation index is determined by subjective weight and objective weight. In this paper, an improved linguistic AHP (L-AHP) method is used to determine the subjective weight, to avoid the problem that the gap between the maximum and the minimum is too large. The L-AHP method is used to determine the subjective weight of the indicator. By using a linguistic term set \{very unimportant, unimportant, slightly unimportant, equal, slightly important, important, very important\}, the comparison matrix between different indexes can be obtained. Through the investigation of 13 experts, the following matrix is constructed as follows (Tables 2-15). 
Table 1. Urban ecosystem health evaluation criteria in Jinan

\begin{tabular}{|c|c|}
\hline Elements & Indicators \\
\hline \multirow{3}{*}{ C1. Vitality } & B1. GDP growth rate \\
\hline & B2. GDP per capita \\
\hline & B3. GDP energy consumption of ten thousand yuan \\
\hline \multirow{9}{*}{$\begin{array}{l}\text { C2. Composition } \\
\text { Structure }\end{array}$} & B4. Forest coverage rate \\
\hline & B5. Per capita public green space in the built-up area \\
\hline & B6. Coverage rate of urban green space \\
\hline & B7. Per capita area of arable land \\
\hline & B8. Proportion of tertiary industry \\
\hline & B9. The proportion of high-tech industry to industrial output \\
\hline & B10. The ratio of R\&D to GDP \\
\hline & B11. Urban population density \\
\hline & B12 The proportion of the aged population \\
\hline \multirow{4}{*}{$\begin{array}{l}\text { C3. Recovery } \\
\text { Capacity }\end{array}$} & B13. Comprehensive utilization of hazardous waste \\
\hline & B14. Urban domestic sewage treatment rate \\
\hline & B15. The comprehensive utilization rate of industrial solid waste \\
\hline & B16. The ratio of environmental investment to GDP \\
\hline \multirow{9}{*}{$\begin{array}{l}\text { C4. The Ecological } \\
\text { System Continually } \\
\text { Offering Service } \\
\text { Function }\end{array}$} & B17. Unemployment rate of urban residents \\
\hline & B18. The number of beds per 10,000 people \\
\hline & B19. Engel's coefficient \\
\hline & B20. Per capita housing area \\
\hline & B21. Annual average value of sulfur dioxide (SO2) \\
\hline & B22. Annual average value of nitrogen dioxide (NO2) \\
\hline & B23. Inhalable particle (PM10) \\
\hline & B24. Precipitation $\mathrm{PH}$ \\
\hline & B25. Good days of air \\
\hline \multirow{4}{*}{$\begin{array}{l}\text { C5. Population } \\
\text { Health }\end{array}$} & B26. Natural population growth rate \\
\hline & B27. Per capita life expectancy \\
\hline & B28. Number of College Students per ten thousand people \\
\hline & B29. Public library collection per hundred people \\
\hline \multirow{5}{*}{$\begin{array}{l}\text { C6. Ecosystem } \\
\text { Cognition }\end{array}$} & B30. Policy completeness for protecting the healthy development of ecosystem \\
\hline & $\begin{array}{l}\text { B31. The degree of local government's attention to healthy development } \\
\text { of ecosystem }\end{array}$ \\
\hline & B32. Ecological protection consciousness of production enterprises \\
\hline & B33. Public concern for ecosystem \\
\hline & B34. Social satisfaction for ecosystem \\
\hline
\end{tabular}


(1) The comparison matrix C

Table 2. The comparison matrix C

\begin{tabular}{|c|c|c|c|c|c|c|}
\hline $\mathrm{A}$ & $\mathrm{C}_{1}$ & $\mathrm{C}_{2}$ & $\mathrm{C}_{3}$ & $\mathrm{C}_{4}$ & $\mathrm{C}_{5}$ & $\mathrm{C}_{6}$ \\
\hline $\mathrm{C}_{1}$ & $\mathrm{~s}_{3}$ & $\mathrm{~s}_{1}$ & $\mathrm{~s}_{3}$ & $\mathrm{~s}_{2}$ & $\mathrm{~s}_{4}$ & $\mathrm{~s}_{3}$ \\
\hline $\mathrm{C}_{2}$ & $\mathrm{~s}_{5}$ & $\mathrm{~s}_{3}$ & $\mathrm{~s}_{5}$ & $\mathrm{~s}_{4}$ & $\mathrm{~s}_{6}$ & $\mathrm{~s}_{5}$ \\
\hline $\mathrm{C}_{3}$ & $\mathrm{~s}_{3}$ & $\mathrm{~s}_{1}$ & $\mathrm{~s}_{3}$ & $\mathrm{~s}_{2}$ & $\mathrm{~s}_{4}$ & $\mathrm{~s}_{3}$ \\
\hline $\mathrm{C}_{4}$ & $\mathrm{~s}_{4}$ & $\mathrm{~s}_{2}$ & $\mathrm{~s}_{4}$ & $\mathrm{~s}_{3}$ & $\mathrm{~s}_{4}$ & $\mathrm{~s}_{4}$ \\
\hline $\mathrm{C}_{5}$ & $\mathrm{~s}_{2}$ & $\mathrm{~s}_{0}$ & $\mathrm{~s}_{2}$ & $\mathrm{~s}_{2}$ & $\mathrm{~s}_{3}$ & $\mathrm{~s}_{2}$ \\
\hline $\mathrm{C}_{6}$ & $\mathrm{~s}_{3}$ & $\mathrm{~s}_{1}$ & $\mathrm{~s}_{3}$ & $\mathrm{~s}_{2}$ & $\mathrm{~s}_{4}$ & $\mathrm{~s}_{3}$ \\
\hline
\end{tabular}

Table 3. The derived matrix of Table 2

\begin{tabular}{|c|c|c|c|c|c|c|}
\hline & $\mathrm{C}_{1}$ & $\mathrm{C}_{2}$ & $\mathrm{C}_{3}$ & $\mathrm{C}_{4}$ & $\mathrm{C}_{5}$ & $\mathrm{C}_{6}$ \\
\hline $\mathrm{C}_{1}$ & 1.000 & 0.513 & 1.000 & 0.717 & 1.396 & 1.000 \\
\hline $\mathrm{C}_{2}$ & 1.948 & 1.000 & 1.948 & 1.396 & 2.718 & 1.948 \\
\hline $\mathrm{C}_{3}$ & 1.000 & 0.513 & 1.000 & 0.717 & 1.396 & 1.000 \\
\hline $\mathrm{C}_{4}$ & 1.396 & 0.717 & 1.396 & 1.000 & 1.396 & 1.396 \\
\hline $\mathrm{C}_{5}$ & 0.717 & 0.368 & 0.717 & 0.717 & 1.000 & 0.717 \\
\hline $\mathrm{C}_{6}$ & 1.000 & 0.513 & 1.000 & 0.717 & 1.396 & 1.000 \\
\hline
\end{tabular}

Thus, the weight is obtained: $\varsigma=(0.142,0.277,0.142,0.188,0.108,0.142)$.

The weights have passed the consistency test.

(2) The comparison matrix $\mathrm{C}_{1}$-B:

Table 4. The comparison matrix $\mathrm{C}_{1}-\mathrm{B}$

Table 5. The derived matrix of Table 4

\begin{tabular}{|c|c|c|c|}
\hline $\mathrm{C}_{1}$ & $\mathrm{~B}_{1}$ & $\mathrm{~B}_{2}$ & $\mathrm{~B}_{2}$ \\
\hline $\mathrm{B}_{1}$ & $\mathrm{~s}_{3}$ & $\mathrm{~s}_{3}$ & $\mathrm{~s}_{2}$ \\
\hline $\mathrm{B}_{2}$ & $\mathrm{~s}_{3}$ & $\mathrm{~s}_{3}$ & $\mathrm{~s}_{2}$ \\
\hline $\mathrm{B}_{3}$ & $\mathrm{~s}_{4}$ & $\mathrm{~s}_{4}$ & $\mathrm{~s}_{3}$ \\
\hline
\end{tabular}

\begin{tabular}{|c|c|c|c|}
\hline $\mathrm{C}_{1}$ & $\mathrm{~B}_{1}$ & $\mathrm{~B}_{2}$ & $\mathrm{~B}_{3}$ \\
\hline $\mathrm{B}_{1}$ & 1.000 & 1.000 & 0.717 \\
\hline $\mathrm{B}_{2}$ & 1.000 & 1.000 & 0.717 \\
\hline $\mathrm{B}_{3}$ & 1.396 & 1.396 & 1.000 \\
\hline
\end{tabular}

Thus, the weight is obtained: $\varsigma_{C_{1}-B}=(0.294,0.294,0.412)$.

The weights have passed the consistency test.

(3) The comparison matrix C2-B

Table 6. The comparison matrix C2-B

\begin{tabular}{|c|c|c|c|c|c|c|c|c|c|}
\hline $\mathrm{C}_{2}$ & $\mathrm{~B}_{4}$ & $\mathrm{~B}_{5}$ & $\mathrm{~B}_{6}$ & $\mathrm{~B}_{7}$ & $\mathrm{~B}_{8}$ & $\mathrm{~B}_{9}$ & $\mathrm{~B}_{10}$ & $\mathrm{~B}_{11}$ & $\mathrm{~B}_{12}$ \\
\hline $\mathrm{B}_{4}$ & $\mathrm{~s}_{3}$ & $\mathrm{~s}_{5}$ & $\mathrm{~s}_{3}$ & $\mathrm{~s}_{3}$ & $\mathrm{~s}_{4}$ & $\mathrm{~s}_{4}$ & $\mathrm{~s}_{3}$ & $\mathrm{~s}_{5}$ & $\mathrm{~s}_{4}$ \\
\hline $\mathrm{B}_{5}$ & $\mathrm{~s}_{1}$ & $\mathrm{~s}_{3}$ & $\mathrm{~s}_{1}$ & $\mathrm{~s}_{1}$ & $\mathrm{~s}_{2}$ & $\mathrm{~s}_{2}$ & $\mathrm{~s}_{1}$ & $\mathrm{~s}_{3}$ & $\mathrm{~s}_{2}$ \\
\hline $\mathrm{B}_{6}$ & $\mathrm{~s}_{3}$ & $\mathrm{~s}_{5}$ & $\mathrm{~s}_{3}$ & $\mathrm{~s}_{3}$ & $\mathrm{~s}_{3}$ & $\mathrm{~s}_{4}$ & $\mathrm{~s}_{4}$ & $\mathrm{~s}_{3}$ & $\mathrm{~s}_{5}$ \\
\hline $\mathrm{B}_{7}$ & $\mathrm{~s}_{3}$ & $\mathrm{~s}_{5}$ & $\mathrm{~s}_{3}$ & $\mathrm{~s}_{3}$ & $\mathrm{~s}_{3}$ & $\mathrm{~s}_{4}$ & $\mathrm{~s}_{4}$ & $\mathrm{~s}_{5}$ & $\mathrm{~s}_{4}$ \\
\hline
\end{tabular}


End of Table 6

\begin{tabular}{|c|c|c|c|c|c|c|c|c|c|}
\hline $\mathrm{C}_{2}$ & $\mathrm{~B}_{4}$ & $\mathrm{~B}_{5}$ & $\mathrm{~B}_{6}$ & $\mathrm{~B}_{7}$ & $\mathrm{~B}_{8}$ & $\mathrm{~B}_{9}$ & $\mathrm{~B}_{10}$ & $\mathrm{~B}_{11}$ & $\mathrm{~B}_{12}$ \\
\hline $\mathrm{B}_{8}$ & $\mathrm{~s}_{2}$ & $\mathrm{~s}_{4}$ & $\mathrm{~s}_{3}$ & $\mathrm{~s}_{3}$ & $\mathrm{~s}_{3}$ & $\mathrm{~s}_{3}$ & $\mathrm{~s}_{3}$ & $\mathrm{~s}_{2}$ & $\mathrm{~s}_{4}$ \\
\hline $\mathrm{B}_{9}$ & $\mathrm{~s}_{2}$ & $\mathrm{~s}_{4}$ & $\mathrm{~s}_{2}$ & $\mathrm{~s}_{2}$ & $\mathrm{~s}_{3}$ & $\mathrm{~s}_{3}$ & $\mathrm{~s}_{3}$ & $\mathrm{~s}_{2}$ & $\mathrm{~s}_{4}$ \\
\hline $\mathrm{B}_{10}$ & $\mathrm{~s}_{3}$ & $\mathrm{~s}_{5}$ & $\mathrm{~s}_{2}$ & $\mathrm{~s}_{2}$ & $\mathrm{~s}_{3}$ & $\mathrm{~s}_{3}$ & $\mathrm{~s}_{3}$ & $\mathrm{~s}_{3}$ & $\mathrm{~s}_{5}$ \\
\hline $\mathrm{B}_{11}$ & $\mathrm{~s}_{1}$ & $\mathrm{~s}_{3}$ & $\mathrm{~s}_{3}$ & $\mathrm{~s}_{1}$ & $\mathrm{~s}_{4}$ & $\mathrm{~s}_{4}$ & $\mathrm{~s}_{3}$ & $\mathrm{~s}_{3}$ & $\mathrm{~s}_{4}$ \\
\hline $\mathrm{B}_{12}$ & $\mathrm{~s}_{2}$ & $\mathrm{~s}_{4}$ & $\mathrm{~s}_{1}$ & $\mathrm{~s}_{2}$ & $\mathrm{~s}_{2}$ & $\mathrm{~s}_{2}$ & $\mathrm{~s}_{1}$ & $\mathrm{~s}_{2}$ & $\mathrm{~s}_{3}$ \\
\hline
\end{tabular}

Table 7. The derived matrix of Table 6

\begin{tabular}{|c|c|c|c|c|c|c|c|c|c|}
\hline $\mathrm{C}_{2}$ & $\mathrm{~B}_{4}$ & $\mathrm{~B}_{5}$ & $\mathrm{~B}_{6}$ & $\mathrm{~B}_{7}$ & $\mathrm{~B}_{8}$ & $\mathrm{~B}_{9}$ & $\mathrm{~B}_{10}$ & $\mathrm{~B}_{11}$ & $\mathrm{~B}_{12}$ \\
\hline $\mathrm{B}_{4}$ & 1.000 & 1.948 & 1.000 & 1.000 & 1.396 & 1.396 & 1.000 & 1.948 & 1.96 \\
\hline $\mathrm{B}_{5}$ & 0.513 & 1.000 & 0.513 & 0.513 & 0.717 & 0.717 & 0.513 & 1.000 & 0.717 \\
\hline $\mathrm{B}_{6}$ & 1.000 & 1.948 & 1.000 & 1.000 & 1.000 & 1.396 & 1.396 & 1.000 & 1.948 \\
\hline $\mathrm{B}_{7}$ & 1.000 & 1.948 & 1.000 & 1.000 & 1.000 & 1.396 & 1.396 & 1.948 & 1.396 \\
\hline $\mathrm{B}_{8}$ & 0.717 & 1.396 & 1.000 & 1.000 & 1.000 & 1.000 & 1.000 & 0.717 & 1.396 \\
\hline $\mathrm{B}_{9}$ & 0.717 & 1.396 & 0.717 & 0.717 & 1.000 & 1.000 & 1.000 & 0.717 & 1.396 \\
\hline $\mathrm{B}_{10}$ & 1.000 & 1.948 & 0.717 & 0.717 & 1.000 & 1.000 & 1.000 & 1.000 & 1.948 \\
\hline $\mathrm{B}_{11}$ & 0.513 & 1.000 & 1.000 & 0.513 & 1.396 & 1.396 & 1.000 & 1.000 & 1.396 \\
\hline $\mathrm{B}_{12}$ & 0.717 & 1.396 & 0.513 & 0.717 & 0.717 & 0.717 & 0.513 & 0.717 & 1.000 \\
\hline
\end{tabular}

Thus, the weight is obtained:

$S_{C_{2}-B}=(0.140,0.072,0.135,0.140,0.109,0.100,0.116,0.107,0.081)$.

The weights have passed the consistency test.

(4) The comparison matrix C3-B

Table 8. The comparison matrix C3-B

\begin{tabular}{|c|c|c|c|c|}
\hline $\mathrm{C}_{3}$ & $\mathrm{~B}_{13}$ & $\mathrm{~B}_{14}$ & $\mathrm{~B}_{15}$ & $\mathrm{~B}_{16}$ \\
\hline $\mathrm{B}_{13}$ & $\mathrm{~s}_{3}$ & $\mathrm{~s}_{4}$ & $\mathrm{~s}_{3}$ & $\mathrm{~s}_{2}$ \\
\hline $\mathrm{B}_{14}$ & $\mathrm{~s}_{2}$ & $\mathrm{~s}_{3}$ & $\mathrm{~s}_{2}$ & $\mathrm{~s}_{1}$ \\
\hline $\mathrm{B}_{15}$ & $\mathrm{~s}_{3}$ & $\mathrm{~s}_{4}$ & $\mathrm{~s}_{3}$ & $\mathrm{~s}_{2}$ \\
\hline $\mathrm{B}_{16}$ & $\mathrm{~s}_{4}$ & $\mathrm{~s}_{5}$ & $\mathrm{~s}_{4}$ & $\mathrm{~s}_{3}$ \\
\hline
\end{tabular}

Table 9. The derived matrix of Table 8

\begin{tabular}{|c|c|c|c|c|}
\hline $\mathrm{C}_{3}$ & $\mathrm{~B}_{13}$ & $\mathrm{~B}_{14}$ & $\mathrm{~B}_{15}$ & $\mathrm{~B}_{16}$ \\
\hline $\mathrm{B}_{13}$ & 1.000 & 1.396 & 1.000 & 0.717 \\
\hline $\mathrm{B}_{14}$ & 0.717 & 1.000 & 0.717 & 0.513 \\
\hline $\mathrm{B}_{15}$ & 1.000 & 1.396 & 1.000 & 0.717 \\
\hline $\mathrm{B}_{16}$ & 1.39 & 1.948 & 1.396 & 1.000 \\
\hline
\end{tabular}

Thus, the weight is obtained: $\varsigma_{C_{3}-B}=(0.243,0.175,0.243,0.339)$.

The weights have passed the consistency test. 
(5) The comparison matrix C4-B

Table 10. The comparison matrix C4-B

\begin{tabular}{|c|c|c|c|c|c|c|c|c|c|}
\hline $\mathrm{C}_{4}$ & $\mathrm{~B}_{17}$ & $\mathrm{~B}_{18}$ & $\mathrm{~B}_{19}$ & $\mathrm{~B}_{20}$ & $\mathrm{~B}_{21}$ & $\mathrm{~B}_{22}$ & $\mathrm{~B}_{23}$ & $\mathrm{~B}_{24}$ & $\mathrm{~B}_{25}$ \\
\hline $\mathrm{B}_{17}$ & $\mathrm{~s}_{3}$ & $\mathrm{~s}_{4}$ & $\mathrm{~s}_{2}$ & $\mathrm{~s}_{3}$ & $\mathrm{~s}_{2}$ & $\mathrm{~s}_{2}$ & $\mathrm{~s}_{2}$ & $\mathrm{~s}_{2}$ & $\mathrm{~s}_{3}$ \\
\hline $\mathrm{B}_{18}$ & $\mathrm{~s}_{2}$ & $\mathrm{~s}_{3}$ & $\mathrm{~s}_{1}$ & $\mathrm{~s}_{2}$ & $\mathrm{~s}_{1}$ & $\mathrm{~s}_{1}$ & $\mathrm{~s}_{1}$ & $\mathrm{~s}_{1}$ & $\mathrm{~s}_{2}$ \\
\hline $\mathrm{B}_{19}$ & $\mathrm{~s}_{4}$ & $\mathrm{~s}_{5}$ & $\mathrm{~s}_{3}$ & $\mathrm{~s}_{4}$ & $\mathrm{~s}_{3}$ & $\mathrm{~s}_{3}$ & $\mathrm{~s}_{3}$ & $\mathrm{~s}_{3}$ & $\mathrm{~s}_{4}$ \\
\hline $\mathrm{B}_{20}$ & $\mathrm{~s}_{3}$ & $\mathrm{~s}_{4}$ & $\mathrm{~s}_{2}$ & $\mathrm{~s}_{3}$ & $\mathrm{~s}_{2}$ & $\mathrm{~s}_{2}$ & $\mathrm{~s}_{2}$ & $\mathrm{~s}_{2}$ & $\mathrm{~s}_{3}$ \\
\hline $\mathrm{B}_{21}$ & $\mathrm{~s}_{4}$ & $\mathrm{~s}_{5}$ & $\mathrm{~s}_{3}$ & $\mathrm{~s}_{4}$ & $\mathrm{~s}_{3}$ & $\mathrm{~s}_{3}$ & $\mathrm{~s}_{3}$ & $\mathrm{~s}_{2}$ & $\mathrm{~s}_{4}$ \\
\hline $\mathrm{B}_{22}$ & $\mathrm{~s}_{4}$ & $\mathrm{~s}_{5}$ & $\mathrm{~s}_{3}$ & $\mathrm{~s}_{4}$ & $\mathrm{~s}_{3}$ & $\mathrm{~s}_{3}$ & $\mathrm{~s}_{3}$ & $\mathrm{~s}_{2}$ & $\mathrm{~s}_{4}$ \\
\hline $\mathrm{B}_{23}$ & $\mathrm{~s}_{4}$ & $\mathrm{~s}_{5}$ & $\mathrm{~s}_{3}$ & $\mathrm{~s}_{4}$ & $\mathrm{~s}_{3}$ & $\mathrm{~s}_{3}$ & $\mathrm{~s}_{3}$ & $\mathrm{~s}_{2}$ & $\mathrm{~s}_{4}$ \\
\hline $\mathrm{B}_{24}$ & $\mathrm{~s}_{4}$ & $\mathrm{~s}_{5}$ & $\mathrm{~s}_{3}$ & $\mathrm{~s}_{4}$ & $\mathrm{~s}_{4}$ & $\mathrm{~s}_{4}$ & $\mathrm{~s}_{4}$ & $\mathrm{~s}_{3}$ & $\mathrm{~s}_{5}$ \\
\hline $\mathrm{B}_{25}$ & $\mathrm{~s}_{3}$ & $\mathrm{~s}_{4}$ & $\mathrm{~s}_{2}$ & $\mathrm{~s}_{3}$ & $\mathrm{~s}_{2}$ & $\mathrm{~s}_{2}$ & $\mathrm{~s}_{2}$ & $\mathrm{~s}_{1}$ & $\mathrm{~s}_{3}$ \\
\hline
\end{tabular}

Table 11. The derived matrix of Table 10

\begin{tabular}{|c|c|c|c|c|c|c|c|c|c|}
\hline $\mathrm{C}_{4}$ & $\mathrm{~B}_{17}$ & $\mathrm{~B}_{18}$ & $\mathrm{~B}_{19}$ & $\mathrm{~B}_{20}$ & $\mathrm{~B}_{21}$ & $\mathrm{~B}_{22}$ & $\mathrm{~B}_{23}$ & $\mathrm{~B}_{24}$ & $\mathrm{~B}_{25}$ \\
\hline $\mathrm{B}_{17}$ & 1.000 & 1.396 & 0.717 & 1.000 & 0.717 & 0.717 & 0.717 & 0.717 & 1.000 \\
\hline $\mathrm{B}_{18}$ & 0.717 & 1.000 & 0.513 & 0.717 & 0.513 & 0.513 & 0.513 & 0.513 & 0.717 \\
\hline $\mathrm{B}_{19}$ & 1.396 & 1.948 & 1.000 & 1.396 & 1.000 & 1.000 & 1.000 & 1.000 & 1.396 \\
\hline $\mathrm{B}_{20}$ & 1.000 & 1.396 & 0.717 & 1.000 & 0.717 & 0.717 & 0.717 & 0.717 & 1.000 \\
\hline $\mathrm{B}_{21}$ & 1.396 & 1.948 & 1.000 & 1.396 & 1.000 & 1.000 & 1.000 & 0.717 & 1.396 \\
\hline $\mathrm{B}_{22}$ & 1.396 & 1.948 & 1.000 & 1.396 & 1.000 & 1.000 & 1.000 & 0.717 & 1.396 \\
\hline $\mathrm{B}_{23}$ & 1.396 & 1.948 & 1.000 & 1.396 & 1.000 & 1.000 & 1.000 & 0.717 & 1.396 \\
\hline $\mathrm{B}_{24}$ & 1.396 & 1.948 & 1.000 & 1.396 & 1.396 & 1.396 & 1.396 & 1.000 & 1.948 \\
\hline $\mathrm{B}_{25}$ & 1.000 & 1.396 & 0.717 & 1.000 & 0.717 & 0.717 & 0.717 & 0.513 & 1.000 \\
\hline
\end{tabular}

Thus, the weight is obtained:

$$
\varsigma_{C_{4}-B}=(0.093,0.067,0.130,0.093,0.125,0.125,0.125,0.152,0.090) \text {. }
$$

The weights have passed the consistency test.

(6) The comparison matrix C5-B

Table 12. The comparison matrix C5-B

\begin{tabular}{|c|c|c|c|c|}
\hline $\mathrm{C}_{5}$ & $\mathrm{~B}_{26}$ & $\mathrm{~B}_{27}$ & $\mathrm{~B}_{28}$ & $\mathrm{~B}_{29}$ \\
\hline $\mathrm{B}_{26}$ & $\mathrm{~s}_{3}$ & $\mathrm{~s}_{3}$ & $\mathrm{~s}_{4}$ & $\mathrm{~s}_{4}$ \\
\hline $\mathrm{B}_{27}$ & $\mathrm{~s}_{3}$ & $\mathrm{~s}_{3}$ & $\mathrm{~s}_{4}$ & $\mathrm{~s}_{4}$ \\
\hline $\mathrm{B}_{28}$ & $\mathrm{~s}_{2}$ & $\mathrm{~s}_{2}$ & $\mathrm{~s}_{3}$ & $\mathrm{~s}_{3}$ \\
\hline $\mathrm{B}_{29}$ & $\mathrm{~s}_{2}$ & $\mathrm{~s}_{2}$ & $\mathrm{~s}_{3}$ & $\mathrm{~s}_{3}$ \\
\hline
\end{tabular}

Table 13. The derived matrix of Table 12

\begin{tabular}{|c|c|c|c|c|}
\hline $\mathrm{C}_{5}$ & $\mathrm{~B}_{26}$ & $\mathrm{~B}_{27}$ & $\mathrm{~B}_{28}$ & $\mathrm{~B}_{29}$ \\
\hline $\mathrm{B}_{26}$ & 1.000 & 1.000 & 1.396 & 1.396 \\
\hline $\mathrm{B}_{27}$ & 1.000 & 1.000 & 1.396 & 1.396 \\
\hline $\mathrm{B}_{28}$ & 1.396 & 1.396 & 1.000 & 1.000 \\
\hline $\mathrm{B}_{29}$ & 1.396 & 1.396 & 1.000 & 1.000 \\
\hline
\end{tabular}

Thus, the weight is obtained: $\varsigma_{C_{5}-B}=(0.291,0.291,0.209,0.209)$.

The weights have passed the consistency test. 
(7) The comparison matrix C6-B

Table 14. The comparison matrix C6-B

\begin{tabular}{|c|c|c|c|c|c|}
\hline $\mathrm{C}_{6}$ & $\mathrm{~B}_{30}$ & $\mathrm{~B}_{31}$ & $\mathrm{~B}_{32}$ & $\mathrm{~B}_{33}$ & $\mathrm{~B}_{34}$ \\
\hline $\mathrm{B}_{30}$ & $\mathrm{~s}_{3}$ & $\mathrm{~s}_{1}$ & $\mathrm{~s}_{2}$ & $\mathrm{~s}_{2}$ & $\mathrm{~s}_{3}$ \\
\hline $\mathrm{B}_{31}$ & $\mathrm{~s}_{5}$ & $\mathrm{~s}_{3}$ & $\mathrm{~s}_{4}$ & $\mathrm{~s}_{4}$ & $\mathrm{~s}_{5}$ \\
\hline $\mathrm{B}_{32}$ & $\mathrm{~s}_{5}$ & $\mathrm{~s}_{2}$ & $\mathrm{~s}_{3}$ & $\mathrm{~s}_{3}$ & $\mathrm{~s}_{4}$ \\
\hline $\mathrm{B}_{33}$ & $\mathrm{~s}_{4}$ & $\mathrm{~s}_{2}$ & $\mathrm{~s}_{3}$ & $\mathrm{~s}_{3}$ & $\mathrm{~s}_{4}$ \\
\hline $\mathrm{B}_{34}$ & $\mathrm{~s}_{3}$ & $\mathrm{~s}_{1}$ & $\mathrm{~s}_{2}$ & $\mathrm{~s}_{2}$ & $\mathrm{~s}_{3}$ \\
\hline
\end{tabular}

Table 15. The derived matrix of Table 14

\begin{tabular}{|c|c|c|c|c|c|}
\hline $\mathrm{C}_{6}$ & $\mathrm{~B}_{30}$ & $\mathrm{~B}_{31}$ & $\mathrm{~B}_{32}$ & $\mathrm{~B}_{33}$ & $\mathrm{~B}_{34}$ \\
\hline $\mathrm{B}_{30}$ & 1.000 & 0.513 & 0.717 & 0.717 & 1.000 \\
\hline $\mathrm{B}_{31}$ & 1.948 & 1.000 & 1.396 & 1.396 & 1.948 \\
\hline $\mathrm{B}_{32}$ & 1.948 & 0.717 & 1.000 & 1.000 & 1.396 \\
\hline $\mathrm{B}_{33}$ & 1.396 & 0.717 & 1.000 & 1.000 & 1.396 \\
\hline $\mathrm{B}_{34}$ & 1.000 & 0.513 & 0.717 & 0.717 & 1.000 \\
\hline
\end{tabular}

Thus, the weight is obtained: $\varsigma_{C_{6}-B}=(0.146,0.285,0.219,0.204,0.146)$.

The weights have passed the consistency test.

Based on the results $\varsigma$ of $\varsigma, \varsigma_{C_{1}-B}, \varsigma_{C_{2}-B}, \varsigma_{C_{3}-B}, \varsigma_{C_{4}-B}, \varsigma_{C_{5}-B}$ and $\varsigma_{C_{6}-B}$, the subjective weights of the 34 indicators are:

$$
\begin{aligned}
\varsigma= & (0.042,0.042,0.058,0.039,0.020,0.037,0.039,0.030,0.028,0.032,0.030,0.022,0.035, \\
& 0.025,0.035,0.048,0.018,0.013,0.024,0.018,0.024,0.024,0.024,0.029,0.017,0.032, \\
& 0.032,0.023,0.023,0.021,0.040,0.031,0.029,0.021) .
\end{aligned}
$$

Therefore, the subjective weights of the 34 indicators are shown in Table 17.

\subsection{Obtain the evaluation values of urban ecosystem in Jinan City}

Those urban ecosystem health quantitative data (B1-B29) of Jinan from 2011 to 2015 are obtained from the yearbook of China's urban statistical yearbook (2012-2016) and Jinan statistical yearbook (2012-2016).

And those qualitative data (B31-B34) are obtained by the grading of 100 related persons. These 100 persons are concluding 20 environmental protection department staff, 20 college staff, 20 enterprise staff, 20 community workers, and 20 environmental enthusiasts. They are invited to use the linguistic set $S=\left\{s_{0}, s_{1}, s_{2}, s_{3}, s_{4}, s_{5}, s_{6}\right\}=\{$ very poor, poor, slightly poor, fair, slightly better, better, best $\}$ to evaluate. In accordance with the relative theories, the scores of 100 persons are converted into probabilistic linguistic after sorting-out in statistics. Taking data of B31 as the example, among 100 persons, 20 persons evaluate fair, 60 persons evaluate slightly better, and 20 persons evaluate better. Then the proportion of choosing fair, slightly better and better is calculated respectively. The data of B31 is expressed as $\{\mathrm{s} 3(0.2), \mathrm{s} 4(0.6), \mathrm{s} 5(0.2)\}$ in probabilistic linguistic. Similarly, the data of B31-B34 from 2011 to 2015 can be obtained. The relevant data are shown in Table 16.

\subsection{Calculate the combined weights of urban ecosystem evaluation indexes in Jinan City}

Step 1: Normalize the decision data.

In this index system, $B_{3}, B_{11}, B_{12}, B_{17}, B_{19}, B_{21}, B_{22}, B_{23}, B_{24}$ are cost indicators, and others are benefit indicators. In order to eliminate the influence of the incommensurability, the data in Table 16 are normalized. According to the formula (1)-(5) introduced in the section 1, the result is shown as follows: 


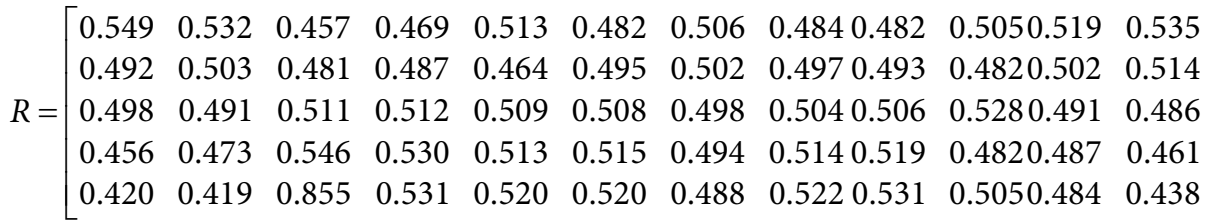

$$
\begin{aligned}
& \begin{array}{llllllllll}
0.512 & 0.491 & 0.498 & 0.702 & 0.364 & 0.262 & 0.541 & 0.365 & 0.482 & 0.501
\end{array} \\
& \begin{array}{lllllllllll}
0.748 & 0.496 & 0.498 & 0.591 & 0.423 & 0.599 & 0.243 & 0.522 & 0.500 & 0.542
\end{array} \\
& \begin{array}{lllllllllll}
0.348 & 0.501 & 0.502 & 0.325 & 0.546 & 0.609 & 0.561 & 0.532 & 0.441 & 0.450
\end{array} \\
& \begin{array}{lllllllllll}
0.238 & 0.511 & 0.502 & 0.231 & 0.624 & 0.449 & 0.578 & 0.558 & 0.569 & 0.501
\end{array} \\
& \begin{array}{lllllllllll}
0.328 & 0.511 & 0.502 & 0.102 & 0.656 & 0.646 & 0.703 & 0.609 & 0.819 & 0.554
\end{array} \\
& 0.533[0.307,0.357] \quad 0.763 \quad 0.503 \quad 0.509 \quad 0.475 \quad 0.540 \quad\left\{s_{3}(0.2), s_{4}(0.6), s_{5}(0.2)\right\} \\
& \begin{array}{llllllll}
0.540 & {[0.301,0.369]} & 0.439 & 0.485 & 0.496 & 0.534 & 0.442 & \left\{s_{4}(0.2), s_{5}(0.8)\right\}
\end{array} \\
& 0.436[0.287,0.336] \quad 0.435 \quad 0.506 \quad 0.497 \quad 0.540 \quad 0.521 \quad\left\{s_{5}(0.7), s_{6}(0.3)\right\}
\end{aligned}
$$

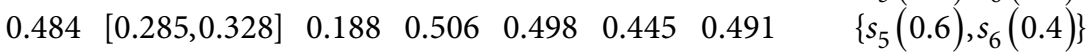

$$
\begin{aligned}
& 0.530[0.277,0.301] \quad 0.201 \quad 0.492 \quad 0.499 \quad 0.445 \quad 0.521 \quad\left\{s_{5}(0.4), s_{6}(0.6)\right\} \\
& \left.\begin{array}{cccc}
\left\{s_{3}(0.3), s_{4}(0.6), s_{5}(0.1)\right\} & \left\{s_{3}(0.3), s_{4}(0.5), s_{5}(0.2)\right\} & \left\{s_{4}(0.5), s_{5}(0.5)\right\} & \left\{s_{3}(0.2), s_{4}(0.6), s_{5}(0.2)\right\} \\
\left\{s_{4}(0.1), s_{5}(0.9)\right\} & \left\{s_{4}(0.4), s_{5}(0.6)\right\} & \left\{s_{4}(0.3), s_{5}(0.7)\right\} & \left\{s_{4}(0.3), s_{5}(0.7)\right\} \\
\left\{s_{5}(0.7), s_{6}(0.3)\right\} & \left\{s_{5}(0.7), s_{6}(0.3)\right\} & \left\{s_{5}(0.7), s_{6}(0.3)\right\} & \left\{s_{5}(0.2), s_{6}(0.8)\right\} \\
\left\{s_{5}(0.6), s_{6}(0.4)\right\} & \left\{s_{5}(0.6), s_{6}(0.4)\right\} & \left\{s_{5}(0.5), s_{6}(0.5)\right\} & \left\{s_{5}(0.3), s_{6}(0.7)\right\} \\
\left\{s_{5}(0.4), s_{6}(0.6)\right\} & \left\{s_{5}(0.2), s_{6}(0.8)\right\} & \left\{s_{5}(0.1), s_{6}(0.9)\right\} & \left\{s_{5}(0.1), s_{6}(0.9)\right\}
\end{array}\right] .
\end{aligned}
$$

Step 2: Calculate the weight of the indicator.

(1) The subjective weight calculated in 4.1 is shown as follows:

$\varsigma=(0.042,0.042,0.058,0.039,0.020,0.037,0.039,0.030,0.028,0.032,0.030,0.022,0.035,0.025$, $0.035,0.048,0.018,0.013,0.024,0.018,0.024,0.024,0.024,0.029,0.017,0.032,0.032,0.023$, $0.023,0.021,0.040,0.031,0.029,0.021)$.

(2) Based on the normalized matrix $R$, the objective weight calculated by formula (11) is shown as follows:

$w=(0.020,0.017,0.057,0.011,0.008,0.006,0.003,0.006,0.008,0.008,0.006,0.016,0.080,0.004$, $0.001,0.104,0.052,0.062,0.064,0.035,0.056,0.016,0.017,0.009,0.092,0.004,0.002,0.019$, $0.015,0.034,0.040,0.046,0.041,0.040)$.

(3) The combination weight calculated by formula (12) is shown as follows $(\alpha=0.4)$ : $\omega=(0.029,0.027,0.058,0.022,0.013,0.019,0.017,0.016,0.016,0.018,0.015,0.019,0.062,0.012$, $0.014,0.082,0.038,0.042,0.048,0.028,0.043,0.019,0.020,0.017,0.062,0.015,0.014,0.020$, $0.018,0.029,0.040,0.040,0.036,0.032$ ).

Therefore, the weights of the 34 indicators are shown in Table 17. 


\begin{tabular}{|c|c|c|c|c|c|c|c|c|c|c|c|c|c|c|c|c|}
\hline 늠 & $\vec{\infty}$ & $\wedge$ & $\begin{array}{l}\stackrel{0}{0} \\
0\end{array}$ & \begin{tabular}{l}
\multirow{H}{*}{} \\
in \\
m
\end{tabular} & $\stackrel{10}{n}$ & $\begin{array}{l}\text { मे } \\
\text { ले }\end{array}$ & $\begin{array}{l}+ \\
\text { İ } \\
\text { in }\end{array}$ & 䚄 & 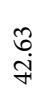 & ĩ & $\mid \begin{array}{l}1 \\
0 \\
n \\
n \\
0\end{array}$ & $\underset{\nearrow}{\beth}$ & $\stackrel{g}{\underline{c}}$ & \& & gे & 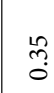 \\
\hline 离 & $\mid \begin{array}{l}\infty \\
\infty\end{array}$ & $\stackrel{9}{\curvearrowright}$ & $\stackrel{\vartheta}{\exists}$ & in & $\stackrel{\rightleftarrows}{=}$ & $\stackrel{\circ}{\dot{m}}$ & $\begin{array}{l}\infty \\
\stackrel{0}{\circ} \\
\text { in }\end{array}$ & 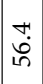 & $\begin{array}{l}0 \\
\vec{F}\end{array}$ & $\vec{i}$ & 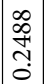 & $\stackrel{b}{=}$ & 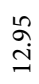 & 8 & & $\stackrel{2}{1}$ \\
\hline
\end{tabular}

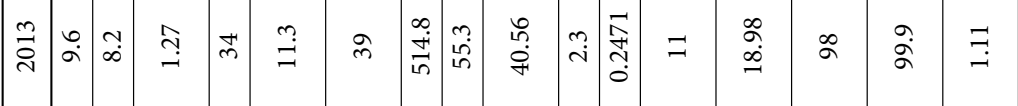

离

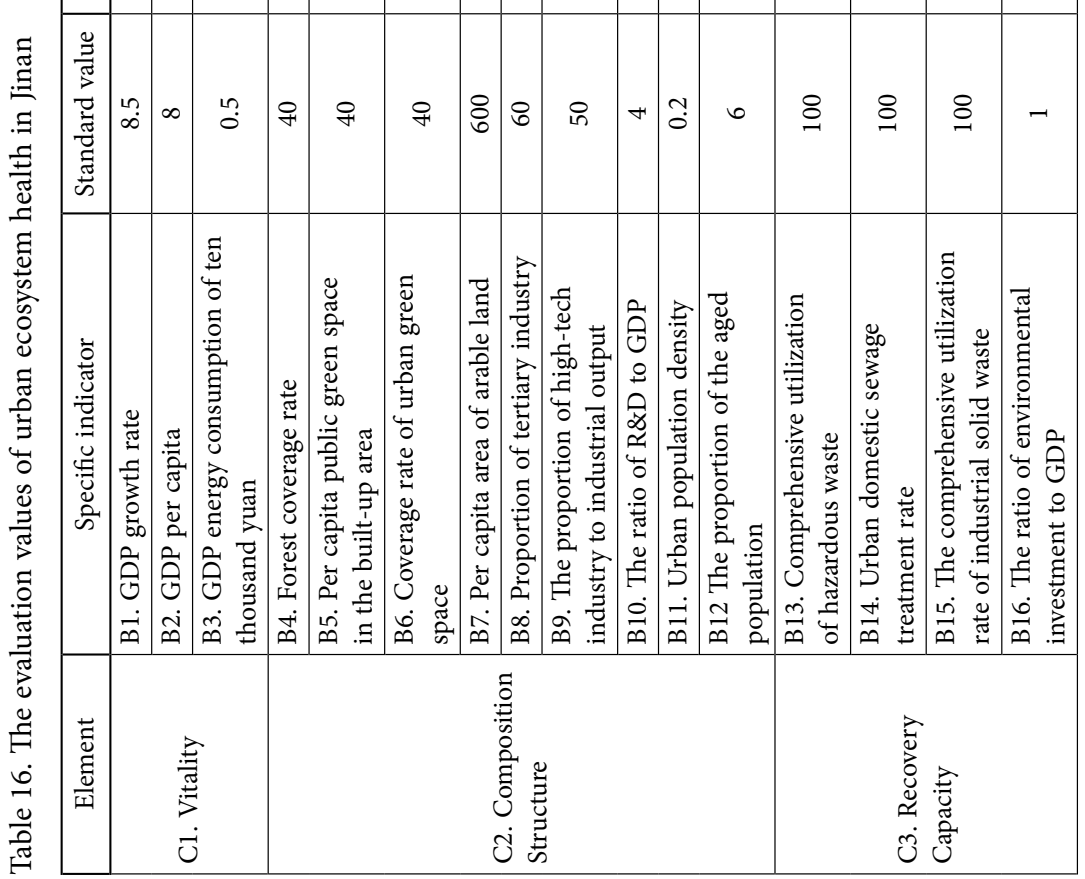




\begin{tabular}{|c|c|c|c|c|c|c|c|c|c|c|c|c|c|c|c|c|c|}
\hline$\stackrel{n}{\stackrel{n}{*}}$ & $\sim$ & $\begin{array}{l}\hat{b} \\
0\end{array}$ & \begin{tabular}{|l|}
$\stackrel{H}{*}$ \\
$\stackrel{N}{N}$
\end{tabular} & $\stackrel{m}{m}$ & $\stackrel{0}{0}$ & $\begin{array}{l}\infty \\
\text { dे } \\
0\end{array}$ & în & 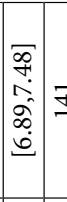 & $\exists \underset{\forall}{\exists}$ & 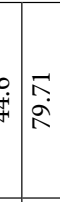 & ڤे & $\begin{array}{l}\stackrel{l}{n} \\
0\end{array}$ & 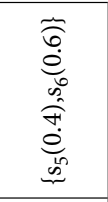 & 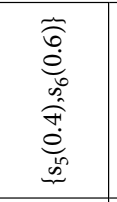 & 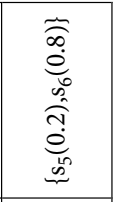 & 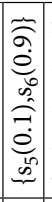 & 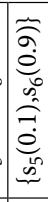 \\
\hline$\stackrel{\sharp}{\stackrel{\sim}{d}}$ & $\vec{i}$ & $\stackrel{\infty}{\stackrel{+}{0}}$ & $\hat{\grave{\lambda}}$ & $\overrightarrow{\vec{i}}$ & $\stackrel{\hat{O}}{\tilde{O}}$ & $\begin{array}{l}\tilde{O} \\
0 \\
0\end{array}$ & $\mid$\begin{tabular}{c}
$\mathbb{N}$ \\
\hdashline \\
0
\end{tabular} & 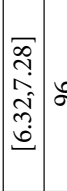 & ঃ & 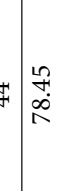 & $\stackrel{n}{n}$ & $\stackrel{n}{n}$ & 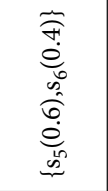 & 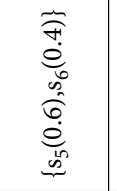 & 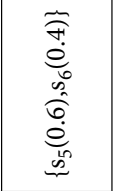 & 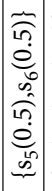 & 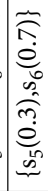 \\
\hline$\stackrel{m}{\stackrel{\sim}{\sim}}$ & $\stackrel{\vec{i}}{\mathrm{i}}$ & $\begin{array}{l}\qquad 0 \\
0 \\
0\end{array}$ & 吕 & $\begin{array}{l}0 \\
\dot{m} \\
\dot{m}\end{array}$ & مे & $\begin{array}{l}\stackrel{\circ}{\hat{0}} \\
0\end{array}$ & $\overrightarrow{\overrightarrow{2}}$ & 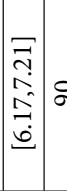 & \& & 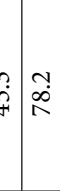 & $\stackrel{n}{R}$ & ?? & 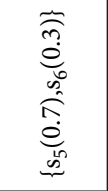 & 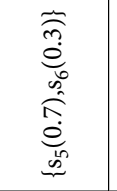 & 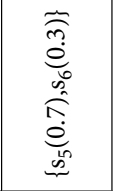 & 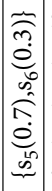 & 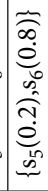 \\
\hline$\stackrel{\sim}{\stackrel{\sim}{\sim}}$ & $\vec{m}$ & $\begin{array}{l}\text { ț } \\
\stackrel{0}{0}\end{array}$ & 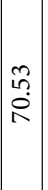 & 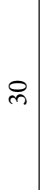 & $\begin{array}{l}\text { Oै } \\
\stackrel{0}{0} \\
0\end{array}$ & $\begin{array}{l}\stackrel{9}{0} \\
\text { Oे }\end{array}$ & 点 & 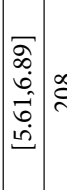 & 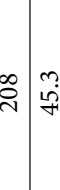 & 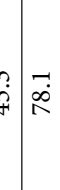 & $\vec{a}$ & ח̂n & 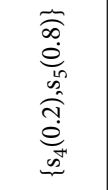 & 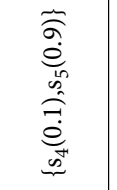 & 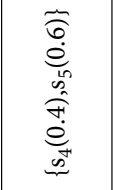 & 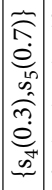 & 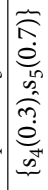 \\
\hline $\overrightarrow{\vec{i}}$ & $\stackrel{0}{\dot{m}}$ & 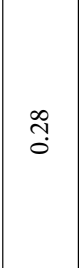 & $\hat{\vec{m}}$ & $\begin{array}{l}\hat{a} \\
\hat{i}\end{array}$ & $\begin{array}{l}n \\
0 \\
0 \\
0 \\
0\end{array}$ & $\begin{array}{l}\hat{n} \\
0 \\
0\end{array}$ & 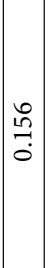 & 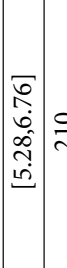 & 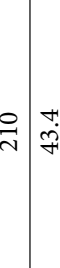 & 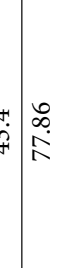 & $a$ & $\stackrel{\text { If }}{\circ}$ & 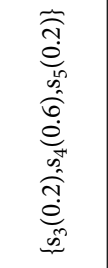 & 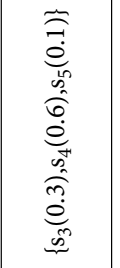 & 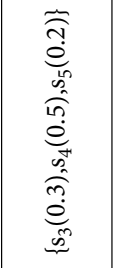 & 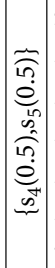 & 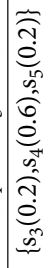 \\
\hline 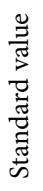 & $\sim$ & $N$ & 尺े & 우 & . & $\stackrel{2}{0}$ & $\stackrel{2}{2}$ & 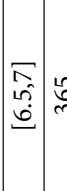 & 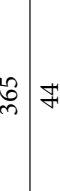 & t) & $\infty$ & $\begin{array}{l}\text { in } \\
\text { ?n }\end{array}$ & 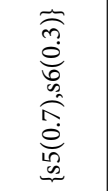 & 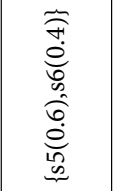 & 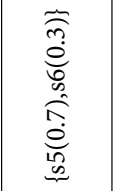 & 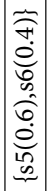 & 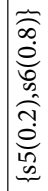 \\
\hline 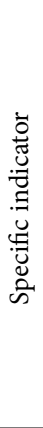 & 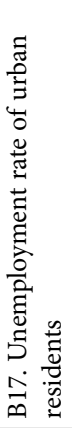 & 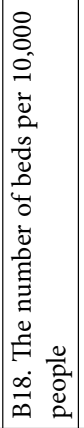 & 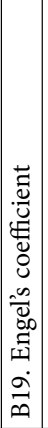 & 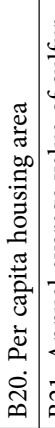 & 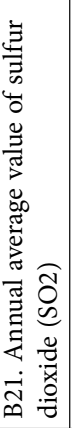 & 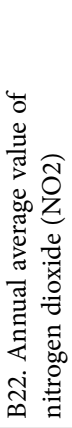 & 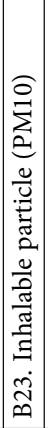 & 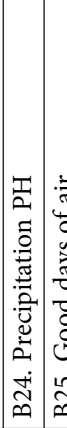 & 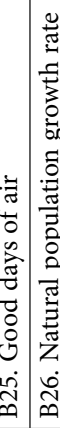 & 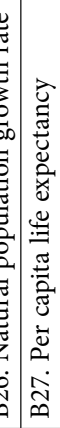 & 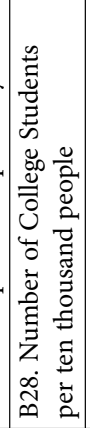 & 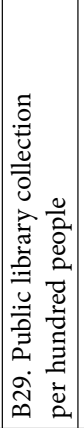 & 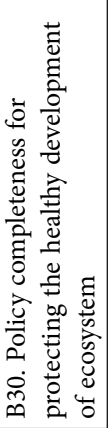 & 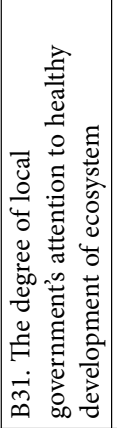 & 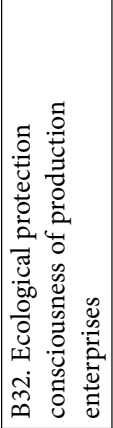 & 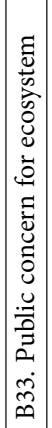 & 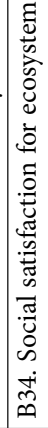 \\
\hline 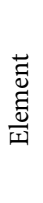 & & & & 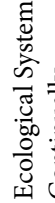 & 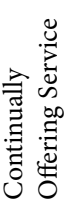 & & & & & & 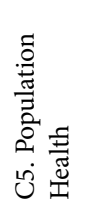 & & & 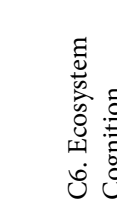 & & & \\
\hline
\end{tabular}


Table 17. The weights of urban ecosystem health index in Jinan

\begin{tabular}{|c|c|c|c|c|c|}
\hline Elements & $\begin{array}{c}\text { Serial } \\
\text { number }\end{array}$ & Specific indicators & $\begin{array}{l}\text { Subjective } \\
\text { weights }\end{array}$ & $\begin{array}{l}\text { Objective } \\
\text { weights }\end{array}$ & $\begin{array}{c}\text { Combination } \\
\text { weights }\end{array}$ \\
\hline \multirow{3}{*}{ C1.Vitality } & $\mathrm{B} 1$ & GDP growth rate & 0.042 & 0.020 & 0.029 \\
\hline & B2 & GDP per capita & 0.042 & 0.017 & 0.027 \\
\hline & B3 & $\begin{array}{l}\text { GDP energy consumption of ten } \\
\text { thousand yuan }\end{array}$ & 0.058 & 0.057 & 0.058 \\
\hline \multirow{9}{*}{$\begin{array}{l}\text { C2. Composi- } \\
\text { tion Structure }\end{array}$} & B4 & Forest coverage rate & 0.039 & 0.011 & 0.022 \\
\hline & B5 & $\begin{array}{l}\text { Per capita public green space in } \\
\text { the built-up area }\end{array}$ & 0.020 & 0.008 & 0.013 \\
\hline & B6 & $\begin{array}{l}\text { Coverage rate of urban green } \\
\text { space }\end{array}$ & 0.037 & 0.006 & 0.019 \\
\hline & B7 & Per capita area of arable land & 0.039 & 0.003 & 0.017 \\
\hline & B8 & Proportion of tertiary industry & 0.030 & 0.006 & 0.016 \\
\hline & B9 & $\begin{array}{l}\text { The proportion of high-tech } \\
\text { industry to industrial output }\end{array}$ & 0.028 & 0.008 & 0.016 \\
\hline & $\mathrm{B} 10$ & The ratio of R\&D to GDP & 0.032 & 0.008 & 0.018 \\
\hline & B11 & Urban population density & 0.030 & 0.006 & 0.015 \\
\hline & B12 & $\begin{array}{l}\text { The proportion of the aged } \\
\text { population }\end{array}$ & 0.022 & 0.016 & 0.019 \\
\hline \multirow{4}{*}{$\begin{array}{l}\text { C3. Recovery } \\
\text { Capacity }\end{array}$} & B13 & $\begin{array}{l}\text { Comprehensive utilization of } \\
\text { hazardous waste }\end{array}$ & 0.035 & 0.080 & 0.062 \\
\hline & B14 & $\begin{array}{l}\text { Urban domestic sewage treatment } \\
\text { rate }\end{array}$ & 0.025 & 0.004 & 0.012 \\
\hline & B15 & $\begin{array}{l}\text { The comprehensive utilization rate } \\
\text { of industrial solid waste }\end{array}$ & 0.035 & 0.001 & 0.014 \\
\hline & B16 & $\begin{array}{l}\text { The ratio of environmental } \\
\text { investment to GDP }\end{array}$ & 0.048 & 0.104 & 0.082 \\
\hline \multirow{9}{*}{$\begin{array}{l}\text { C4. The } \\
\text { Ecological } \\
\text { System } \\
\text { Continually } \\
\text { Offering } \\
\text { Service } \\
\text { Function }\end{array}$} & B17 & $\begin{array}{l}\text { Unemployment rate of urban } \\
\text { residents }\end{array}$ & 0.018 & 0.052 & 0.038 \\
\hline & B18 & $\begin{array}{l}\text { The number of beds per } 10,000 \\
\text { people }\end{array}$ & 0.013 & 0.062 & 0.042 \\
\hline & B19 & Engel's coefficient & 0.024 & 0.064 & 0.048 \\
\hline & B20 & Per capita housing area & 0.018 & 0.035 & 0.028 \\
\hline & B21 & $\begin{array}{l}\text { Annual average value of sulfur } \\
\text { dioxide (SO2) }\end{array}$ & 0.024 & 0.056 & 0.043 \\
\hline & B22 & $\begin{array}{l}\text { Annual average value of nitrogen } \\
\text { dioxide (NO2) }\end{array}$ & 0.024 & 0.016 & 0.019 \\
\hline & B23 & Inhalable particle (PM10) & 0.024 & 0.017 & 0.020 \\
\hline & B24 & Precipitation $\mathrm{PH}$ & 0.029 & 0.009 & 0.017 \\
\hline & B25 & Good days of air & 0.017 & 0.092 & 0.062 \\
\hline
\end{tabular}


End of Table 17

\begin{tabular}{|c|c|c|c|c|c|}
\hline Elements & $\begin{array}{c}\text { Serial } \\
\text { number }\end{array}$ & Specific indicators & $\begin{array}{c}\text { Subjective } \\
\text { weights }\end{array}$ & $\begin{array}{l}\text { Objective } \\
\text { weights }\end{array}$ & $\begin{array}{c}\text { Combination } \\
\text { weights }\end{array}$ \\
\hline \multirow{4}{*}{$\begin{array}{l}\text { C5. Popula- } \\
\text { tion Health }\end{array}$} & B26 & Natural population growth rate & 0.032 & 0.004 & 0.015 \\
\hline & B27 & Per capita life expectancy & 0.032 & 0.002 & 0.014 \\
\hline & B28 & $\begin{array}{l}\text { Number of College Students per } \\
\text { ten thousand people }\end{array}$ & 0.023 & 0.019 & 0.020 \\
\hline & B29 & $\begin{array}{l}\text { Public library collection per } \\
\text { hundred people }\end{array}$ & 0.023 & 0.015 & 0.018 \\
\hline \multirow{5}{*}{$\begin{array}{l}\text { C6. Ecosys- } \\
\text { tem Cogni- } \\
\text { tion }\end{array}$} & B30 & $\begin{array}{l}\text { Policy completeness for protecting } \\
\text { the healthy development of } \\
\text { ecosystem }\end{array}$ & 0.021 & 0.034 & 0.029 \\
\hline & B31 & $\begin{array}{l}\text { The degree of local government's } \\
\text { attention to healthy development } \\
\text { of ecosystem }\end{array}$ & 0.040 & 0.040 & 0.040 \\
\hline & B32 & $\begin{array}{l}\text { Ecological protection } \\
\text { consciousness of production } \\
\text { enterprises }\end{array}$ & 0.031 & 0.046 & 0.040 \\
\hline & B33 & Public concern for ecosystem & 0.029 & 0.041 & 0.036 \\
\hline & B34 & Social satisfaction for ecosystem & 0.021 & 0.040 & 0.032 \\
\hline
\end{tabular}

\subsection{Use extended TODIM method to evaluate the health status of urban ecosystem in Jinan City}

The relative weight of indicator calculated by formula (13) is shown as follows:

$$
\begin{aligned}
\omega^{\prime}= & (0.353,0.331,0.707,0.237,0.155,0.213,0.192,0.197,0.215,0.187,0.230,0.760,0.149,0.176, \\
& 1.000,0.470,0.516,0.589,0.343,0.530,0.237,0.243,0.204,0.763,0.183,0.169,0.247,0.222, \\
& 0.352,0.495,0.493,0.444,0.393)
\end{aligned}
$$

Calculate the overall dominance of the alternative by formula (14) and formula (15) $(\theta=1)$, the result is shown as following:

$$
\delta\left(A_{i}, A_{k}\right)=\left[\begin{array}{ccccc}
0 & -2.269 & -2.830 & -2.825 & -3.509 \\
0.014 & 0 & -2.341 & -2.265 & -3.089 \\
0.130 & 0.186 & 0 & -0.869 & -1.894 \\
0.214 & -0.147 & -0.526 & 0 & -1.863 \\
0.445 & 0.377 & -0.044 & 0.418 & 0
\end{array}\right] .
$$

To sum up, according to the TODIM method, the ranking of Jinan ecosystem health from 2011 to 2015 can be obtained respectively: $\xi_{1}=0, \xi_{2}=0.339, \xi_{3}=0.814, \xi_{4}=0.822, \xi_{5}=1$, from the result: $2015>2014>2013>2012>2011$. That is, the ecological health in 2015 is the best, and the ecological health in 2011 is the worst.

To further verifying the stability of the evaluation results about the urban ecosystem health in Jinan, we take different parameter values to calculate this case and get the ranking results as shown in Table 18. It is found from Table 4 and Figure 1 that the ranking results are 
consistent when the loss attenuation coefficient takes different value. Although the ranking results of the calculation are consistent, the TODIM method, based on the prospect theory, fully considers the risk aversion attitude of the decision-makers, and can reflect the risk preference of the decision-makers through the adjustment of the parameters, which is more in line with the actual decision requirements.

Table 18. Impact of parameter $\theta$ on decision results

\begin{tabular}{|c|c|c|c|c|c|c|c|c|}
\hline Alternative & \multicolumn{2}{|c|}{$\theta=1$} & \multicolumn{2}{|c|}{$\theta=1.5$} & \multicolumn{2}{|c|}{$\theta=2$} & \multicolumn{2}{|c|}{$\theta=2.25$} \\
\hline year & $\xi_{i}$ & order & $\xi_{i}$ & order & $\xi_{i}$ & order & $\xi_{i}$ & order \\
\hline 2011 & $\xi_{1}=0$ & 5 & $\xi_{1}=0$ & 5 & $\xi_{1}=0$ & 5 & $\xi_{1}=0$ & 5 \\
\hline 2012 & $\xi_{2}=0.339$ & 4 & $\xi_{2}=0.337$ & 4 & $\xi_{2}=0.335$ & 4 & $\xi_{2}=0.335$ & 4 \\
\hline 2013 & $\xi_{3}=0.814$ & 3 & $\xi_{3}=0.809$ & 3 & $\xi_{3}=0.804$ & 3 & $\xi_{3}=0.802$ & 3 \\
\hline 2014 & $\xi_{4}=0.822$ & 2 & $\xi_{4}=0.817$ & 2 & $\xi_{4}=0.812$ & 2 & $\xi_{4}=0.810$ & 2 \\
\hline 2015 & $\xi_{5}=1$ & 1 & $\xi_{5}=1$ & 1 & $\xi_{5}=1$ & 1 & $\xi_{5}=1$ & 1 \\
\hline \multirow{2}{*}{$\begin{array}{c}\text { Alternative } \\
\text { year }\end{array}$} & \multicolumn{2}{|c|}{$\theta=2.5$} & \multicolumn{2}{|c|}{$\theta=8$} & \multicolumn{2}{|c|}{$\theta=10$} & \multicolumn{2}{|c|}{$\theta=14$} \\
\hline & $\xi_{i}$ & order & $\xi_{i}$ & order & $\xi_{i}$ & order & $\xi_{i}$ & order \\
\hline 2011 & $\xi_{1}=0$ & 5 & $\xi_{1}=0$ & 5 & $\xi_{1}=0$ & 5 & $\xi_{1}=0$ & 5 \\
\hline 2012 & $\xi_{2}=0.333$ & 4 & $\xi_{2}=0.314$ & 4 & $\xi_{2}=0.308$ & 4 & $\xi_{2}=0.297$ & 4 \\
\hline 2013 & $\xi_{3}=0.800$ & 3 & $\xi_{3}=0.753$ & 3 & $\xi_{3}=0.738$ & 3 & $\xi_{3}=0.712$ & 3 \\
\hline 2014 & $\xi_{4}=0.808$ & 2 & $\xi_{4}=0.762$ & 2 & $\xi_{4}=0.747$ & 2 & $\xi_{4}=0.721$ & 2 \\
\hline 2015 & $\xi_{5}=1$ & 1 & $\xi_{5}=1$ & 1 & $\xi_{5}=1$ & 1 & $\xi_{5}=1$ & 1 \\
\hline
\end{tabular}

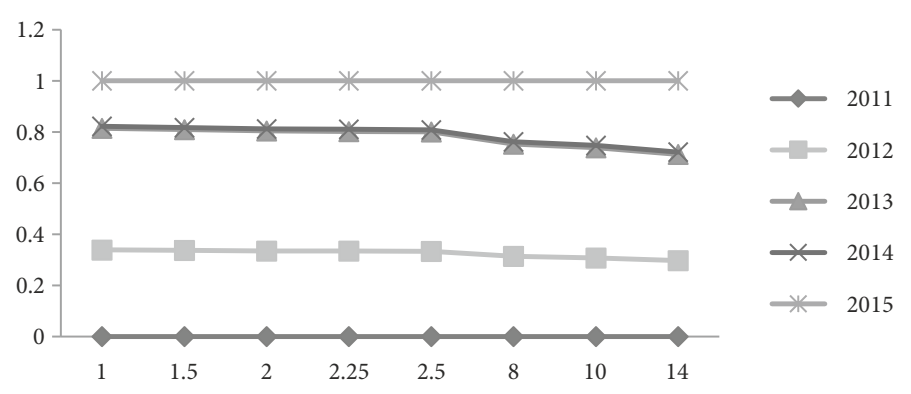

Figure 1. The overall prospect values with different parameter $\theta$

\subsection{Use health index method to calculate the health status of urban ecosystem in Jinan}

(1) According to the standard value of each indicator, that is, the index is normalized to 1 . We have completed the index of completion in the past 5 years $(0-1.0)$. The completion degree formula is shown as following: 
If $z_{i j}$ is real number

$$
y_{i j}= \begin{cases}\min \left(\frac{z_{i j}}{z_{j}^{*}}, 1\right) & j \in I_{1} \\ \min \left(\frac{z_{j}^{*}}{z_{i j}}, 1\right) & j \in I_{2}\end{cases}
$$

$z_{j}^{*}$ is the standard value under each indicator.

If $z_{i j}=\left[b_{i j}^{l}, b_{i j}^{r}\right]$ is interval number,

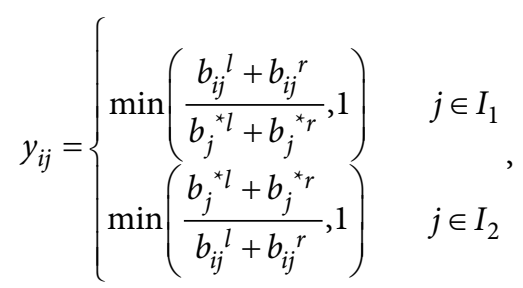

$z_{j}^{*}=\left[b_{i j}^{*}, b_{i j}^{*}\right]$ is the standard value under each indicator.

If $z_{i j}=\left\{L_{i j}^{(d)}\left(p_{i j}^{(d)}\right) \mid d=1,2, \ldots, \# z_{i j}\right\}$ is probabilistic linguistic term set (PLTS),

$$
\begin{aligned}
y_{i j}=\left\{\begin{array}{c}
\min \left(\frac{S\left(z_{i j}\right)}{S\left(z_{j}^{*}\right)}, 1\right) \\
\min \left(\frac{S\left(z_{j}^{*}\right)}{S\left(z_{i j}\right)}, 1\right) \quad j \in I_{1}
\end{array},\right. \\
S\left(z_{i j}\right)=\sum_{d=1}^{\# Z_{i j}} r_{i j}^{(d)} p_{i j}^{(d)} / \sum_{d=1}^{\# Z_{i j}} p_{i j}^{(d)} ; \\
S\left(z_{j}^{*}\right)=\sum_{d=1}^{\# Z_{j}^{*}} r_{j}^{*(d)} p_{j}^{*(d)} / \sum_{d=1}^{\# Z_{j}^{*}} p_{j}^{*(d)},
\end{aligned}
$$

$z_{j}^{*}=\left\{L_{j}^{*(d)}\left(p_{j}^{*(d)}\right) \mid d=1,2, \ldots, \# z_{j}^{*}\right\}$ is the standard value under each indicator.

$\# Z_{i j}$ is the number of linguistic terms in probabilistic linguistic $Z_{i j}$, \# $Z_{j}^{*}$ is the number of linguistic terms in probabilistic linguistic $Z_{j}^{*}$.

Therefore, the degree assignment matrix $Y=\left[y_{i j}\right]_{m \times n}$ can be realized, as shown in Table 19.

(2) Calculate the combined weighted value, $Z=Y \times \omega^{T}$.

The weight is obtained by the combined weight obtained in the article, and the calculation can be obtained: $Z_{1}=0.712, Z_{2}=0.741, Z_{3}=0.755, Z_{4}=0.757, Z_{5}=0.761$.

(3) The judgment of urban ecosystem health status. The health status of urban ecosystem is divided into 5 grades: health, sub-health, vulnerability, disease and bad. The value is nonequidistant, and the corresponding values to health level are respectively: $[0.9,1.0],[0.7,0.9]$, $[0.5,0.7],[0.3,0.5],[0.0,0.3]$. The relationship between comprehensive value and health is shown in Table 20. 
Table 19. Assigned values of urban ecosystem health in Jinan

\begin{tabular}{|c|c|c|c|c|c|c|c|}
\hline $\begin{array}{c}\text { Serial } \\
\text { number }\end{array}$ & Indicators & $\begin{array}{l}\text { Standard } \\
\text { values }\end{array}$ & 2011 & 2012 & 2013 & 2014 & 2015 \\
\hline B1 & GDP growth rate & 8.5 & 1.000 & 1.000 & 1.000 & 1.000 & 0.953 \\
\hline B2 & GDP per capita & 8 & 1.000 & 1.000 & 1.000 & 0.988 & 0.875 \\
\hline B3 & $\begin{array}{l}\text { GDP energy consumption of ten } \\
\text { thousand yuan }\end{array}$ & 0.5 & 0.352 & 0.370 & 0.394 & 0.420 & 0.658 \\
\hline B4 & Forest coverage rate & 40 & 0.778 & 0.808 & 0.850 & 0.880 & 0.881 \\
\hline B5 & $\begin{array}{l}\text { Per capita public green space in the } \\
\text { built-up area }\end{array}$ & 40 & 0.285 & 0.258 & 0.283 & 0.285 & 0.289 \\
\hline B6 & Coverage rate of urban green space & 40 & 0.925 & 0.950 & 0.975 & 0.990 & 0.999 \\
\hline B7 & Per capita area of arable land & 600 & 0.873 & 0.865 & 0.858 & 0.851 & 0.841 \\
\hline B8 & Proportion of tertiary industry & 60 & 0.885 & 0.908 & 0.922 & 0.940 & 0.953 \\
\hline B9 & $\begin{array}{l}\text { The proportion of high-tech } \\
\text { industry to industrial output }\end{array}$ & 50 & 0.773 & 0.791 & 0.811 & 0.832 & 0.853 \\
\hline $\mathrm{B} 10$ & The ratio of R\&D to GDP & 4 & 0.550 & 0.525 & 0.575 & 0.525 & 0.550 \\
\hline B11 & Urban population density & 0.2 & 0.857 & 0.827 & 0.809 & 0.804 & 0.799 \\
\hline B12 & $\begin{array}{l}\text { The proportion of the aged } \\
\text { population }\end{array}$ & 6 & 0.600 & 0.577 & 0.545 & 0.517 & 0.492 \\
\hline B13 & $\begin{array}{l}\text { Comprehensive utilization of } \\
\text { hazardous waste }\end{array}$ & 100 & 0.279 & 0.408 & 0.190 & 0.130 & 0.179 \\
\hline B14 & $\begin{array}{l}\text { Urban domestic sewage treatment } \\
\text { rate }\end{array}$ & 100 & 0.960 & 0.970 & 0.980 & 1.000 & 1.000 \\
\hline B15 & $\begin{array}{l}\text { The comprehensive utilization rate } \\
\text { of industrial solid waste }\end{array}$ & 100 & 0.990 & 0.990 & 0.999 & 0.999 & 0.999 \\
\hline B16 & $\begin{array}{l}\text { The ratio of environmental } \\
\text { investment to GDP }\end{array}$ & 1 & 1.000 & 1.000 & 1.000 & 0.79 & 0.35 \\
\hline B17 & $\begin{array}{l}\text { Unemployment rate of urban } \\
\text { residents }\end{array}$ & 2 & 0.556 & 0.645 & 0.833 & 0.952 & 1.000 \\
\hline B18 & $\begin{array}{l}\text { The number of beds per } 10,000 \\
\text { people }\end{array}$ & 2 & 0.140 & 0.320 & 0.325 & 0.240 & 0.345 \\
\hline B19 & Engel's coefficient & 30 & 0.946 & 0.425 & 0.980 & 1.000 & 1.000 \\
\hline $\mathrm{B} 20$ & Per capita housing area & 40 & 0.524 & 0.750 & 0.765 & 1.000 & 1.000 \\
\hline B21 & $\begin{array}{l}\text { Annual average value of sulfur } \\
\text { dioxide (SO2) }\end{array}$ & 0.05 & 0.588 & 0.610 & 0.538 & 0.694 & 1.000 \\
\hline B22 & $\begin{array}{l}\text { Annual average value of nitrogen } \\
\text { dioxide }(\mathrm{NO} 2)\end{array}$ & 0.05 & 0.943 & 1.000 & 0.847 & 0.943 & 1.000 \\
\hline $\mathrm{B} 23$ & Inhalable particle (PM10) & 0.15 & 0.962 & 0.974 & 0.785 & 0.872 & 0.955 \\
\hline B24 & Precipitation $\mathrm{PH}$ & {$[6.5,7]$} & 1.000 & 1.000 & 1.000 & 0.993 & 0.939 \\
\hline B25 & Good days of air & 365 & 0.575 & 0.570 & 0.247 & 0.263 & 0.386 \\
\hline B26 & Natural population growth rate & 44 & 0.986 & 1.000 & 1.000 & 1.000 & 1.000 \\
\hline B27 & Per capita life expectancy & 80 & 0.973 & 0.976 & 0.978 & 0.981 & 0.996 \\
\hline
\end{tabular}


End of Table 19

\begin{tabular}{|c|l|c|c|c|c|c|c|}
\hline $\begin{array}{c}\text { Serial } \\
\text { number }\end{array}$ & \multicolumn{1}{|c|}{ Indicators } & $\begin{array}{c}\text { Standard } \\
\text { values }\end{array}$ & 2011 & 2012 & 2013 & 2014 & 2015 \\
\hline B28 & $\begin{array}{l}\text { Number of College Students per } \\
\text { ten thousand people }\end{array}$ & 8 & 1.000 & 1.000 & 0.938 & 0.938 & 0.738 \\
\hline B29 & $\begin{array}{l}\text { Public library collection per } \\
\text { hundred people }\end{array}$ & 0.55 & 0.818 & 0.964 & 0.909 & 0.964 & 1.000 \\
\hline B30 & $\begin{array}{l}\text { Policy completeness for protecting } \\
\text { the healthy development of } \\
\text { ecosystem }\end{array}$ & $\left\{s_{5}(0.7), \mathrm{s}_{6}(0.3)\right\}$ & 0.755 & 0.906 & 1.000 & 1.000 & 1.000 \\
\hline B31 & $\begin{array}{l}\text { The degree of local government's } \\
\text { attention to healthy development } \\
\text { of ecosystem }\end{array}$ & $\left\{\mathrm{s}_{5}(0.6), \mathrm{s}_{6}(0.4)\right\}$ & 0.704 & 0.907 & 0.981 & 1.000 & 1.000 \\
\hline B32 & $\begin{array}{l}\text { Ecological protection consciousness } \\
\text { of production enterprises }\end{array}$ & $\left\{\mathrm{s}_{5}(0.7), \mathrm{s}_{6}(0.3)\right\}$ & 0.736 & 0.868 & 1.000 & 1.000 & 1.000 \\
\hline B33 & Public concern for ecosystem & $\left\{\mathrm{s}_{5}(0.6), \mathrm{s}_{6}(0.4)\right\}$ & 0.833 & 0.870 & 0.981 & 1.000 & 1.000 \\
\hline B34 & Social satisfaction for ecosystem & $\left\{\mathrm{s}_{5}(0.2), \mathrm{s}_{6}(0.8)\right\}$ & 0.690 & 0.810 & 1.000 & 0.983 & 1.000 \\
\hline
\end{tabular}

Table 20. Classification standards of urban ecosystem health

\begin{tabular}{|l|c|c|c|c|c|}
\hline \multicolumn{1}{|c|}{ Health status } & Health & Sub-health & Vulnerability & Disease & Bad \\
\hline Health grade & I & II & III & IV & V \\
\hline Comprehensive range of values & {$[0.9,1.0]$} & {$[0.7,0.9)$} & {$[0.5,0.7)$} & {$[0.3,0.5)$} & {$[0.0,0.3]$} \\
\hline
\end{tabular}

From above, the health status of urban ecosystem in Jinan, 2011-2015 years, is shown in Table 21.

Table 21. Comprehensive urban ecosystem health index in Jinan from 2011 to 2015

\begin{tabular}{|c|c|c|c|}
\hline Year & Comprehensive value & Health grade & Health status \\
\hline 2011 & 0.721 & II & Sub-health \\
\hline 2012 & 0.741 & II & Sub-health \\
\hline 2013 & 0.755 & II & Sub-health \\
\hline 2014 & 0.757 & II & Sub-health \\
\hline 2015 & 0.761 & II & Sub-health \\
\hline
\end{tabular}

Based on the above empirical evaluation and analysis, we can conclude that in recent 5 years, the health status of Jinan's urban ecosystem is not optimistic, and the effect of improvement is not obvious. From Table 21, we can see that the health status of the urban ecosystem in Jinan has been wandering in sub-health, and there is still a long way to go from the healthy urban ecosystem. 


\section{Analysis on the changes of urban ecosystem health in Jinan}

To further analysis of each index effecting evaluation index system, health index was used to analyze the trend of each index from 2011 to 2015 . To set 2011 data to 1 , data between 2012 and 2015 is relative values: for the benefit index data of each year, the original data are divided by the original data of 2011; for the cost index data (B3, B11, B12, B17, B19, B21, B22, B23, B24) of each year, the original data of 2011 were divided by the original data of the year.

As can be seen from Table 21, the healthy state of the urban ecosystem in Jinan has been developing towards a positive direction and the index kept growing slightly from 0.721 in 2011 to 0.761 in 2015 . From Figure 2, the ecosystem services of Jinan city were continuously enhanced, the cognition of the ecosystem was steadily improved, and the vitality remained stable at around 1 from 2011 to 2014, it was obvious that it increased sharply to 1.331 in 2015, which was the similar trend of the ecological system continually offering service function. On the contrary, the recovery capacity experienced a slow climb from 2011 to 2012 and declined significantly from 1.179 to 0.463 after 2012 .

From Figure 3, the trend of unit GDP energy consumption kept growing, which meant unit GDP energy consumption decreased in original data. And it was noticeable that the increase of vitality depended on the continual decline of unit GDP energy consumption. The decline of GDP growth rate and GDP per capita showed that Jinan's economic development slowed down, which meant that the two indicators could hardly influence city ecosystem. Therefore, the increase of vitality was determined by the reduction of GDP energy consumption.

From Figure 4, three specific indicators have witnessed the consistent growth. High-tech industry, urban green space, and proportion of tertiary industry all experienced the above constant increase, which became the main driver of composition structure. Forest coverage rate continuously rose from 2011 to 2014 and remained at the highest level between 2014 and 2015. Among those indictors, the rapid increase of the aged population should draw government's attention, because the high proportion of the aged population will do harm to the ecosystem health of the Jinan.

From Figure 5, the rate of comprehensive utilization of industrial solid waste and the rate of urban domestic sewage treatment have remained stable from 2011 to 2015, which have a

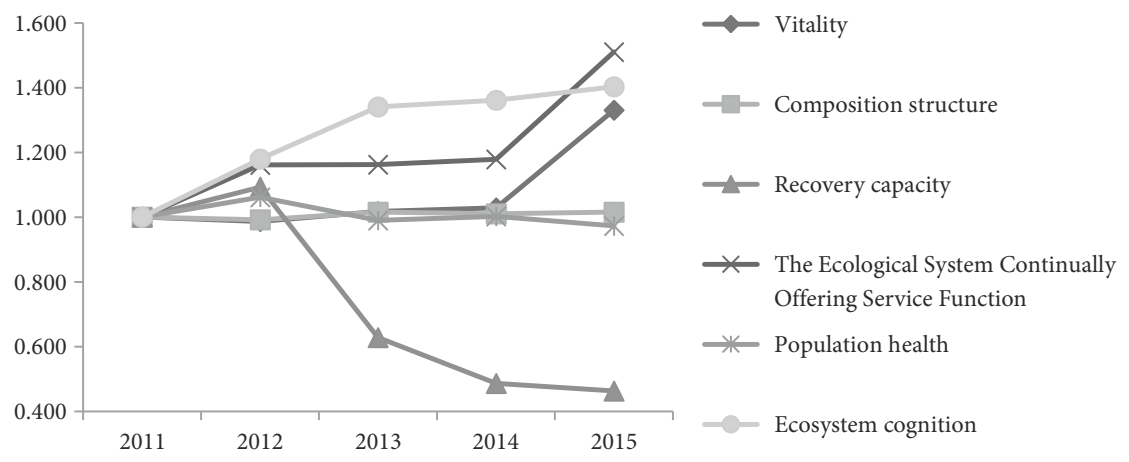

Figure 2. Curves of urban ecosystem health indexes (C1-C6) in Jinan 


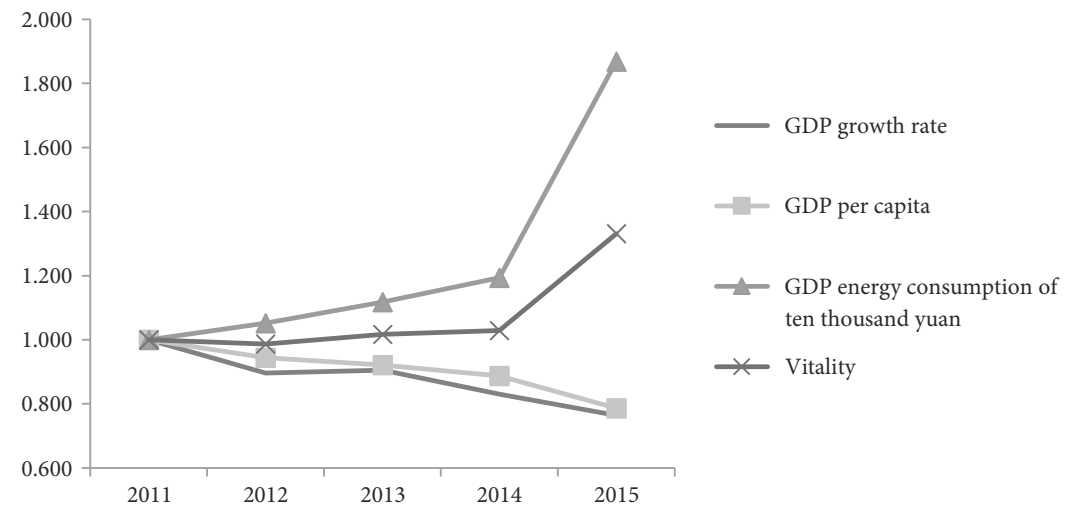

Figure 3. Curves of Vitality element (C1) and specific indicators (B1-B3)
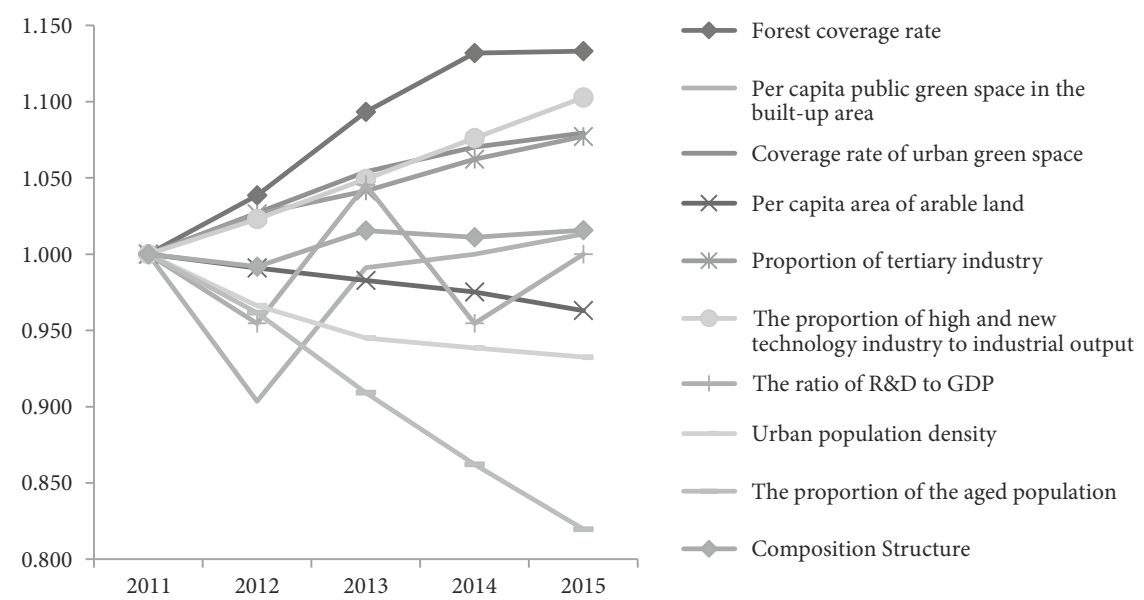

Figure 4. Curves of Composition Structure element (C2) and specific indicators (B4-B12)

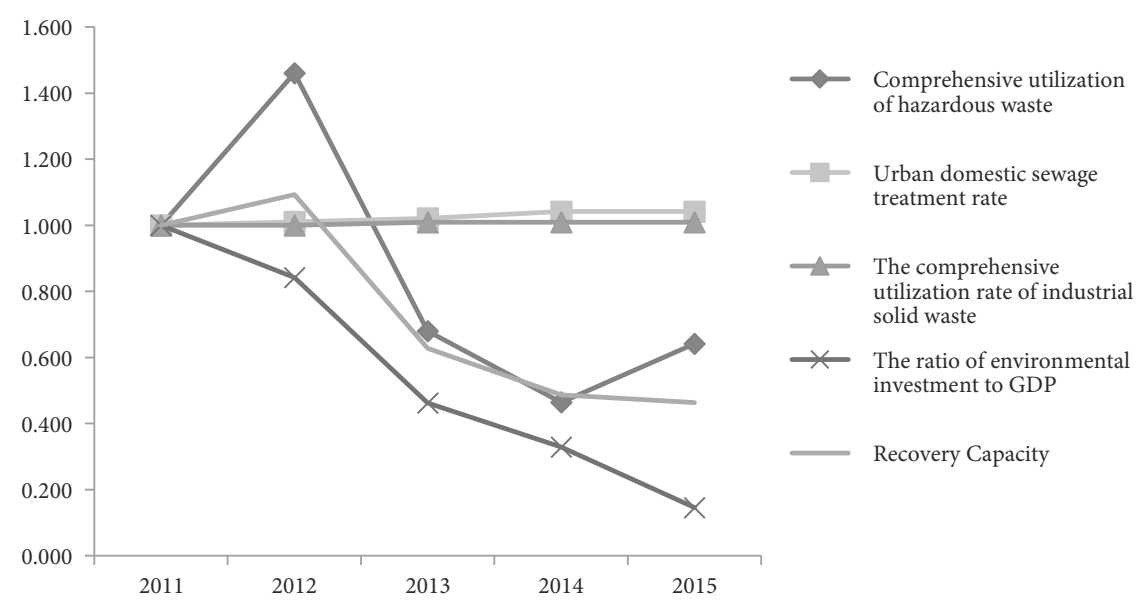

Figure 5. Curves of Recovery Capacity (C3) and specific indicators (B13-B16) 
positive effect on the ecosystem health. However, the ratio of environmental investment to GDP decreased significantly from 2011 to 2015, which led to the negative effect on recovery capacity. Therefore, the weak recovery capacity was caused by the slight ratio of environmental investment to GDP.

From Figure 6, the ecological system continually offering service function shows an increasing trend. This trend was mainly caused by the specific indicator, the number of beds per 10000 people, which jumped from 2011 to 2012 and reached the highest point at 2015. On the contrary, the air condition had got worse. The average value of PM10 and NO2 slightly increased after fluctuation. It is obvious that good days of air reduced sharply after 2012, which indicated the minimum value among specific indicators.

From Figure 7, population health remained almost unchanged from 2011 to 2015. Among the specific indicators, natural population growth rate and per capita life expectancy leveled off. Public library collection per hundred people had an increasing trend, which showed the top value at 2015. While the number of college students per ten thousand people went down
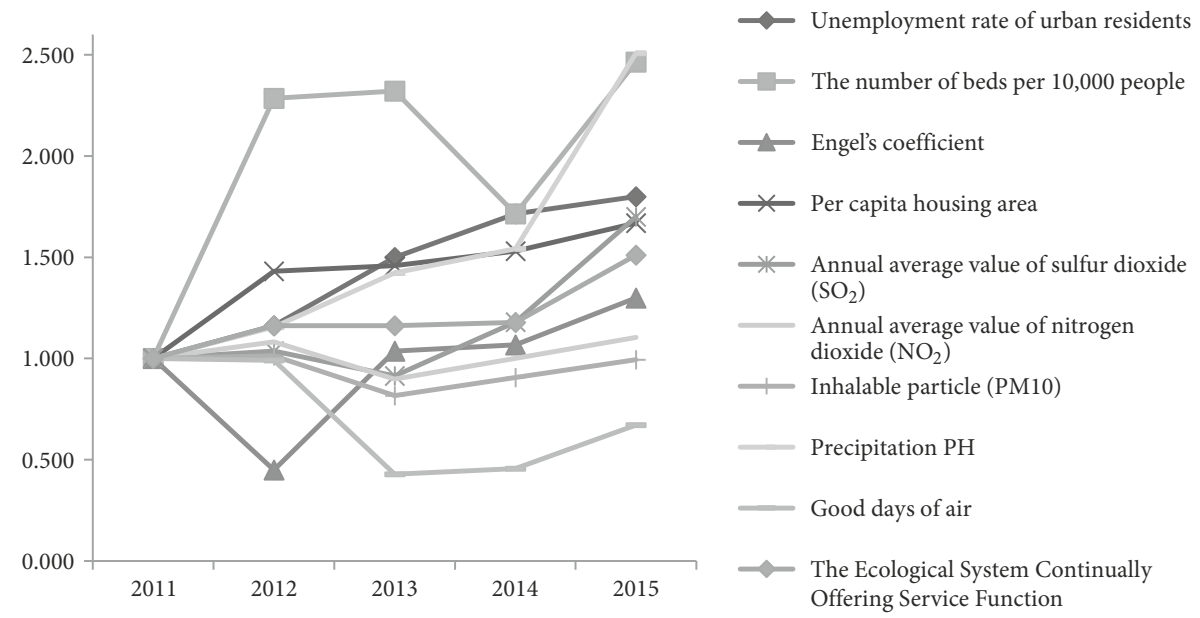

Figure 6. Curves of The Ecological System Continually Offering Service Function (C4) and specific indicators (B17-B25)

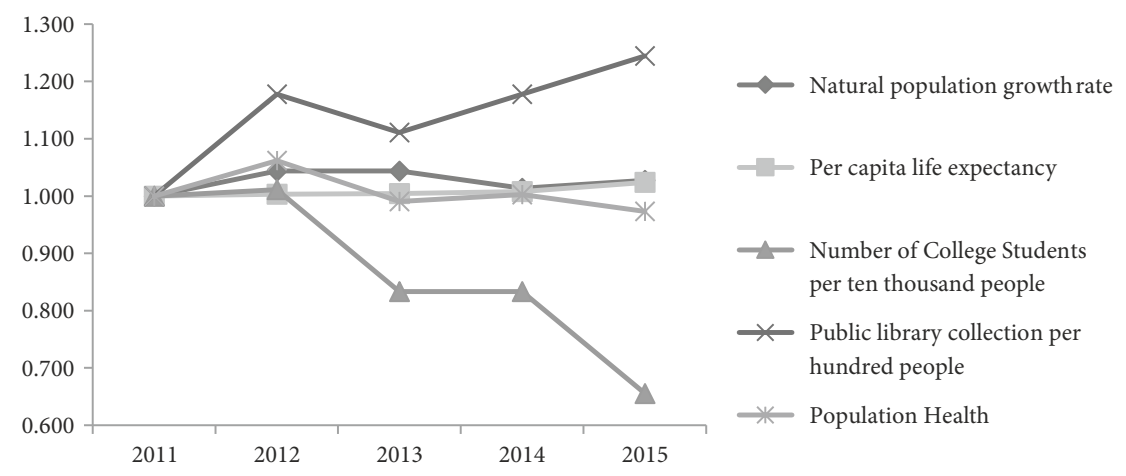

Figure 7. Curves of Population Health (C5) and specific indicators (B26-B29) 
step by step after 2012, which indicated the bottom value at 2015. It was the number of college students per ten thousand people that stopped the increasing trend of population health.

From Figure 8, ecosystem cognition had seen an obvious increase. All specific indicators rose from 2011, except ecological protection consciousness of production enterprises, which dropped a little after 2014. Public concern for ecosystem was the only indicator that climbed slower. Its highest value at 2015 just equaled the other value (1.3) at 2013. So, public concern for ecosystem needs developing in the future.

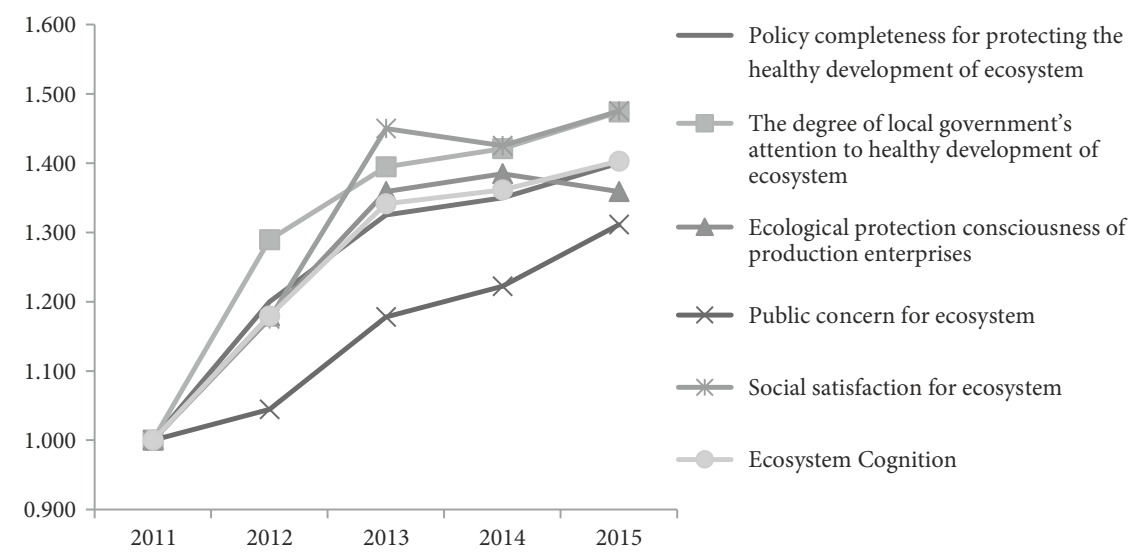

Figure 8. Curves of Ecosystem Cognition element (C6) and specific indicators (B30-B34)

In summary, the advantage elements have included the vitality, the ecological system continually offering service function, and ecosystem cognition. By contrast, the disadvantages elements referred to recovery capacity, composition structure, and population health. In the element of recovery capacity, the weakness of indicator mainly reflected in the low ratio of environmental investment to GDP, which decreased significantly and was harmful for the healthy development of the urban ecological system. In the element of composition structure, the drawback indicator reflected in high proportion of the aged population. In the element of population health, the number of college students per ten thousand people can be marked as the negative indicator. Besides, among the advantage element of vitality, two indicators, GDP growth rate and GDP per capita, had been constantly decreased which delayed the increase of vitality; among the element of ecosystem cognition, the indicator, ecological protection consciousness of production enterprises, slowed down the improvement of ecosystem cognition. All the influential indicators mentioned above can be optimized by three aspects, the governments, the enterprises, and the public.

\section{Conclusions}

Urban ecosystem health is the main driver for promoting the construction of urban ecological civilization. This paper is proposed to evaluate the health status of Jinan city ecosystem, based on the evaluation index system of city ecosystem health and with combination 
weighting method to determine index weight, which overcome the shortcomings of dilemma between actual importance and objective weighing method, and the subjective weighting method. Therefore, extended TODIM method is proposed based on the hybrid index, to evaluate the health status of Jinan city ecosystem. The results of the evaluation show that from 2011 to 2015, the health of urban ecosystems in Jinan has been developing in a better direction, but the environmental quality is worrying. Furthermore, we use the health index method to analyze each index's role in the healthy development of the urban ecosystem one by one, finding out the disadvantages in the healthy development of the urban ecosystem, and put forward the appropriate development countermeasures as following.

(1) Governments should focus on enhancing environmental investment to GDP and optimizing proportion of the aged population.

On one hand, governments should invest more on environmental protection, take measures to improve environmental quality. When pursuing economic interests, governments should pay more attention to environmental protection and enhance environmental investment, which makes Jinan city ecosystem more energetic. On the other hand, governments should go on optimizing proportion of the aged population. In the contemporary society, aged population has become the obstacle of city development. Jinan Government should carry out corresponding measures to encourage citizens to raise more babies. In the longterm, with the increase of young work force, the composition structure can be optimized, which is beneficial to Jinan city ecosystem and makes Jinan stronger competitiveness in the economic development.

(2) Enterprises should focus on enhancing their awareness of ecological protection and offer more job opportunity.

For one thing, the health of Jinan ecosystem has been affected by the discharge of pollutants such as nitrogen dioxide, sulfur dioxide and inhalable particles, which requires enterprises to follow the national policy on energy conservation, emission reduction, and green production. With the principle of minimum use of resources and minimum impact on environment, enterprises should improve their awareness of ecological protection. For another, enterprises should focus on the expansion in the new field. With cultivation of talents and the innovation of science and technology, enterprises can provide more work opportunity, which will lead to the increase of GDP growth rate and GDP per capita.

(3) The public should focus on strengthening the consciousness of birth and the consciousness of education.

The former consciousness is that the public should increase the birth rate of population, so as to reduce the proportion of aged population. Only if the growth of population in Jinan could adapt to the rapid and healthy development of urban economy and society, Jinan ecosystem could make the harmonious development among population, resources and environment. The latter consciousness is that the public should improve the quality of the population, which mainly reflects on the increasing proportion to receive higher education. The number of college students per ten thousand people is an important indicator. The more adults receive further education, the more achievement can be fulfilled by the cultivation of human resources. To achieve the above factors could provide the important intellectual and technical support for the ecological development of Jinan. 


\section{Acknowledgements}

This work is supported by the National Natural Science Foundation of China (Nos. 71771140 and 71471172), the Special Funds of Taishan Scholars Project of Shandong Province (No. ts201511045), Shandong Provincial Social Science Planning Project (Nos. 17BGLJ04, 16CGLJ31 and 16CKJJ27), and Key research and development program of Shandong Province (2016GNC110016).

\section{References}

Bayram, A., Önsoy, H., Bulut, V. N., \& Akinci, G. (2013). Influences of urban wastewaters on the stream water quality: a case study from Gumushane Province. Turkey, Environmental Monitoring and Assessment, 185(2), 1285-1303. https://doi.org/10.1007/s10661-012-2632-y

Bell, M. L., Cifuentes, L. A., Davis, D. L., Cushing, E., Telles, A. G., \& Gouveia, N. (2011). Environmental health indicators and a case study of air pollution in Latin American cities. Environmental Research, 111(1), 57-66. https://doi.org/10.1016/j.envres.2010.10.005

Berberoglu, S. (2003). Sustainable management for the eastern Mediterranean coast of Turkey. Environmental Management, 31(3), 0442-0451.

Brevik, E. C., Pereg, L., Pereira, P., Steffan, J. J., Burgess, L. C., \& Gedeon, C. I. (2019). Shelter, clothing, and fuel: Often overlooked links between soils, ecosystem services, and human health. Science of the Total Environment, 651, 134-142. https://doi.org/10.1016/j.scitotenv.2018.09.158

Brussard, P. F., Reed, J. M., \& Tracy, C. R. (1998). Ecosystem management: what is it really? Landscape and Urban Planning, 40(1), 9-20. https://doi.org/10.1016/S0169-2046(97)00094-7

Derkzen, M. L., Teeffelen, A. J., \& Verburg, P. H. (2015). Quantifying urban ecosystem services based on high-resolution data of urban green space: an assessment for Rotterdam, the Netherlands. Journal of Applied Ecology, 52(4), 1020-1032. https://doi.org/10.1111/1365-2664.12469

Dou, X., Li, S., \& Wang, J. (2013). Ecological strategy of city sustainable development. APCBEE Procedia, 5, 429-434. https://doi.org/10.1016/j.apcbee.2013.05.074

du Preez, G. C., Daneel, M. S., Wepener, V., \& Fourie, H. (2018). Beneficial nematodes as bioindicators of ecosystem health in irrigated soils. Applied Soil Ecology, 132, 155-168.

https://doi.org/10.1016/j.apsoil.2018.08.008

Fan, Z. P., Zhang, X., Chen, F. D., \& Liu, Y. (2013). Extended TODIM method for hybrid multiple attribute decision making problems. Knowledge-Based Systems, 42(2), 40-48. https://doi.org/10.1016/j.knosys.2012.12.014

Gentile, J. H., Harwell, M. A., Jr, W. C., Harwell, C. C., Deangelis, D., Davis, S., et al. (2001). Ecological conceptual models: a framework and case study on ecosystem management for south Florida sustainability. Science of the Total Environment, 274(1-3), 231-253. https://doi.org/10.1016/S0048-9697(01)00746-X

Gomes, L. F. A. M., \& Lima, M. M. P. P. (1991). TODIM: basics and application to multicriteria ranking of projects with environmental impacts. Foundations of Computing and Decision Sciences, 16(4), 113-127.

Gomes, L. F. A. M., \& Rangel, L. A. D. (2009). An application of the TODIM method to the multicriteria rental evaluation of residential properties. European Journal of Operational Research, 193(1), 204-211. https://doi.org/10.1016/j.ejor.2007.10.046

Guan, H. J., Sun, W. K., \& Zhao, A. W. (2016). Hybrid MADM based on the Extended VIKOR method and its application to ecosystem health evaluation. Journal of Residuals Science \& Technology, 13(8), 113.1-113.8. 
Haeuber, R. (1998). Ecosystem management and environmental policy in the united states: open window or closed door? Landscape and Urban Planning, 40(1-3), 221-233. https://doi.org/10.1016/S0169-2046(97)00115-1

He, Y., Hao, J. Y., He, W., Lam, K. C., \& Xu, F. L. (2019). Spatiotemporal variations of aquatic ecosystem health status in Tolo Harbor, Hong Kong from 1986 to 2014. Ecological Indicators, 100, 20-29. https://doi.org/10.1016/j.ecolind.2018.04.056

Huang, J., Li, Z., \& Liu, H. C. (2017). New approach for failure mode and effect analysis using linguistic distribution assessments and TODIM method. Reliability Engineering \& System Safety, 167, 302-309. https://doi.org/10.1016/j.ress.2017.06.014

Huang, Y. H., \& Wei, G. W. (2018). TODIM method for Pythagorean 2-tuple linguistic multiple attribute decision making. Journal of Intelligent \& Fuzzy Systems, 35(1), 901-915. https://doi.org/10.3233/JIFS-171636

Jiang, Y., Liang, X., \& Liang, H. (2017). An I-TODIM method for multi-attribute decision making with interval numbers. Soft Computing, 21(18), 5489-5506. https://doi.org/10.1007/s00500-016-2139-5

Lackey, R. T. (1998). Seven pillars of ecosystem management 1. Landscape and Urban Planning, 40(1-3), 21-30. https://doi.org/10.1016/S0169-2046(97)00095-9

Li, F., Ye, Y., Song, B., \& Wang, R. (2014). Evaluation of urban suitable ecological land based on the minimum cumulative resistance model: a case study from Changzhou, China. Ecological Modelling, 318(1), 194-203.

Li, Y., \& Liu, P. (2015). Some Heronian mean operators with 2-tuple linguistic information and their application to multiple attribute group decision making. Technological and Economic Development of Economy, 21(5), 797-814. https://doi.org/10.3846/20294913.2015.1055614

Liu, G. Y., Yang, Z. F., Chen, B., Zhang, Y., Zhang, L. X., Zhao, Y. W., \& Jiang, M. M. (2009). Emergybased urban ecosystem health assessment: A case study of Baotou, China. Communications in Nonlinear Science \& Numerical Simulation, 14(3), 972-981. https://doi.org/10.1016/j.cnsns.2007.09.017

Liu, P. (2009). Study on evaluation methods and application of enterprise informatization level based on fuzzy multi-attribute decision making ( $\mathrm{PhD}$ Thesis). Beijing Jiaotong University, China.

Liu, P. (2017). Multiple attribute group decision making method based on interval-valued intuitionistic fuzzy power Heronian aggregation operators. Computers \& Industrial Engineering, 108, 199-212. https://doi.org/10.1016/j.cie.2017.04.033

Liu, P., \& Chen, S. M. (2017). Group decision making based on Heronian aggregation operators of intuitionistic fuzzy numbers. IEEE Transactions on Cybernetics, 47(9), 2514-2530. https://doi.org/10.1109/TCYB.2016.2634599

Liu, P., \& Chen, S. M. (2018). Multiattribute group decision making based on intuitionistic 2-Tuple linguistic information. Information Sciences, 430-431, 599-619. https://doi.org/10.1016/j.ins.2017.11.059

Liu, P., Chen, S. M., \& Liu, J. (2017a). Some intuitionistic fuzzy interaction partitioned Bonferroni mean operators and their application to multi-attribute group decision making. Information Sciences, 411, 98-121. https://doi.org/10.1016/j.ins.2017.05.016

Liu, P., \& Li, H. (2017). Interval-valued intuitionistic fuzzy power Bonferroni aggregation operators and their application to group decision making. Cognitive Computation, 9(4), 494-512. https://doi.org/10.1007/s12559-017-9453-9

Liu, P., Liu, J., \& Chen, S. M. (2018a). Some intuitionistic fuzzy dombi bonferroni mean operators and their application to multi-attribute group decision making. Journal of the Operational Research Society, 69(1), 1-24. https://doi.org/10.1057/s41274-017-0190-y

Liu, P., Liu, J., \& Merigó, J. M. (2018b). Partitioned Heronian means based on linguistic intuitionistic fuzzy numbers for dealing with multi-attribute group decision making. Applied Soft Computing, 62, 395-422. https://doi.org/10.1016/j.asoc.2017.10.017 
Liu, P., Teng, F., Chu, Y., \& Guan, H. (2017b). Health evaluation of urban ecosystem in Shandong Province. China University of Petroleum press.

Liu, P., \& Wang, P. (2018). Some q-Rung orthopair fuzzy aggregation operators and their applications to multiple-attribute decision making. International Journal of Intelligent Systems, 33(2), 259-280. https://doi.org/10.1002/int.21927

Llamazares, B. (2018). An analysis of the generalized TODIM method. European Journal of Operational Research, 269(3), 1041-1049. https://doi.org/10.1016/j.ejor.2018.02.054

Lourenzutti, R., Krohling, R. A., \& Reformat, M. Z. (2017). Choquet based TOPSIS and TODIM for dynamic and heterogeneous decision making with criteria interaction. Information Sciences, 408, 41-69. https://doi.org/10.1016/j.ins.2017.04.037

Mahdi, A., Hosseini, A., Pourahmad, A., \& Hataminejad, H. (2016). Analysis of effective environmental factors an urban health, a case study of Qom, Iran. Habitat International, 55, 89-99. https://doi.org/10.1016/j.habitatint.2016.03.001

Mishra, A. R., \& Rani, P. (2018). Biparametric information measures-based TODIM technique for interval-valued intuitionistic fuzzy environment. Arabian Journal for Science and Engineering, 43(6), 3291-3309. https://doi.org/10.1007/s13369-018-3069-6

Noss, R. F. (2000). High-risk ecosystems as foci for considering biodiversity and ecological integrity in ecological risk assessments. Environmental Science \& Policy, 3(6), 321-332. https://doi.org/10.1016/S1462-9011(00)00112-X

Pang, Q., Wang, H., \& Xu, Z. (2016). Probabilistic linguistic term sets in multi-attribute group decision making. Information Sciences, 369, 128-143. https://doi.org/10.1016/j.ins.2016.06.021

Pereira, J. (2013). Robustness analysis in a TODIM-based multicriteria evaluation model of rental properties. Technological \& Economic Development of Economy, 19(sup1), S176-S190.

Salomon, V. A. P., \& Rangel, L. A. D. (2015). Comparing rankings from using TODIM and a fuzzy expert system. Procedia Computer Science, 55, 126-138. https://doi.org/10.1016/j.procs.2015.07.019

Spiegel, J. M., Bonet, M., Yassi, A., Molina, E., Concepcion, M., \& Mast, P. (2001). Developing ecosystem health indicators in Centro Habana: a community-based approach. Ecosystem Health, 7(1), 15-26. https://doi.org/10.1046/j.1526-0992.2001.007001015.x

van Heezik, Y., \& Brymer, E. (2018). Nature as a commodity: what's good for human health might not be good for ecosystem health. Frontiers in Psychology, 9, 1-16. https://doi.org/10.3389/fpsyg.2018.01673

Wu, Y. N., Wang, J., Hu, Y., Ke, Y. M., Li, L. W. Y. (2018). An extended TODIM-PROMETHEE method for waste-to-energy plant site selection based on sustainability perspective. Energy, 156, 1-16. https://doi.org/10.1016/j.energy.2018.05.087 